\title{
GRADIENT-BASED STRUCTURAL CHANGE DETECTION FOR NONSTATIONARY TIME SERIES M-ESTIMATION
}

\author{
BY WeICHI Wu AND ZHOU ZhOU ${ }^{1}$ \\ University College London and University of Toronto
}

\begin{abstract}
We consider structural change testing for a wide class of time series Mestimation with nonstationary predictors and errors. Flexible predictor-error relationships, including exogenous, state-heteroscedastic and autoregressive regressions and their mixtures, are allowed. New uniform Bahadur representations are established with nearly optimal approximation rates. A CUSUMtype test statistic based on the gradient vectors of the regression is considered. In this paper, a simple bootstrap method is proposed and is proved to be consistent for M-estimation structural change detection under both abrupt and smooth nonstationarity and temporal dependence. Our bootstrap procedure is shown to have certain asymptotically optimal properties in terms of accuracy and power. A public health time series dataset is used to illustrate our methodology, and asymmetry of structural changes in high and low quantiles is found.
\end{abstract}

1. Introduction. Consider the following stochastic linear regression:

$$
y_{i}=\mathbf{x}_{i}^{\prime} \beta+e_{i}
$$

where $\left\{\mathbf{x}_{i}\right\}_{i=1}^{n}$ and $\left\{e_{i}\right\}_{i=1}^{n}$ are the $p$-dimensional predictor time series and error series, respectively. We estimate the unknown parameter vector $\beta$ by an M-estimator $\hat{\beta}_{n}$ :

$$
\hat{\beta}_{n}=\underset{\beta}{\operatorname{argmin}} \sum_{i=1}^{n} \rho\left(y_{i}-\mathbf{x}_{i}^{\prime} \beta\right),
$$

where $\rho(\cdot)$ is a convex loss function with left derivative $\psi(\cdot)$. By choosing different loss functions $\rho$, (1) contains a wide class of frequently used regression models. For instance, for a pre-specified $\tau \in(0,1), \hat{\beta}_{n}$ is the estimate of the $\tau$ th quantile regression coefficient if we set $\rho(x)=\tau x^{+}+(1-\tau)(-x)^{+}$with the left derivative $\psi(x)=\tau-\mathbf{1}(x \leq 0)$. Other important examples include expectile regression with $\rho(x)=|\mathbf{1}(x \leq 0)-\alpha| x^{2}, 0<\alpha<1$, robust $\mathcal{L}_{q}$ regression with $\rho(x)=|x|^{q}, 1<$ $q<2$, the Huber's estimate with $\rho(x)=x^{2} \mathbf{1}(|x| \leq \varsigma) / 2+\left(\varsigma|x|-\varsigma^{2} / 2\right) \mathbf{1}(|x|>$ $\varsigma), \varsigma>0$ and the least squares estimate with $\rho(x)=x^{2}$.

Received August 2016; revised February 2017.

${ }^{1}$ Supported in part by NSERC of Canada.

MSC2010 subject classifications. 62J20, 62M10, 62G09, 62G10.

Key words and phrases. M-estimation, piecewise local stationarity, bootstrap, structural change. 
The purpose of this paper is to provide a theoretical foundation as well as a unified methodological tool for the inference of (1) with a wide class of nonstationary predictor and error processes. For brevity and clarity, we will focus on the structural change detection problem for model (1) throughout this article. Various other results such as confidence region construction and goodness-of-fit tests can be easily established as corollaries of those provided in this paper. The most significant contributions of the paper lie in the following two aspects. First, we investigate the behaviors of a wide class of stochastic M-estimators and their residual processes under a general nonlinear and nonstationary time series framework with a very flexible modeling of the relationship between the regressors and errors. On one hand, following [32], we allow the regressors $\left\{\mathbf{x}_{i}\right\}$ and the errors $\left\{e_{i}\right\}$ to experience both smooth and abrupt nonlinear changes in their marginal distributions as well as dependence structures over time. Such nonlinear and nonstationary modeling of the regressors and errors could be realistic and flexible in many time series applications; see, for instance, the Hong Kong public health time series analyzed in Section 5. On the other hand, by carefully choosing the filtration (information) that generates the predictor and error processes, we are able to provide a unified treatment for a wide class of predictor-error relationships, including exogenous, state-heteroscedastic and autoregressive regressions and their mixtures. Here, "state-heteroscedasticity" refers to probabilistic dependence between the errors and covariates. Equivalently, it represents that, conditional on the covariates, the distribution of the error at any fixed time changes with respect to different levels of the covariates. Under the aforementioned settings, we are able to establish a uniform Bahadur representation of the partial sample M-estimators with nearly optimal approximation rates and derive the limiting behaviors of a gradient-based structural change test. Our theoretical development depends heavily on investigating the conditional empirical processes of M-estimators of dependent and heteroscedastic data. In particular, both martingale and conditional chaining techniques are used to investigate the maximum stochastic oscillations of the conditional gradient processes. Then the maximum stochastic oscillations of the unconditional empirical processes are recovered by certain integration techniques. To our knowledge, this paper provides the first theoretical investigation into general stochastic M-estimations under time series nonstationarity.

Second, we propose in this paper a unified bootstrap methodology, which is consistent for structural change tests of a wide class of M-estimations under both abruptly and smoothly time-varying temporal dynamics and predictor-error dependence. To our knowledge, there have been no methodological results on structural change tests for time series M-estimation with nonstationary covariates and errors in the literature. For change point tests of the mean, [32] proposed a bootstrap procedure which is robust to general forms of nonstationarity in the time series. However, it is highly nontrivial to extend such bootstrap procedures to gradient change point tests for M-estimations. In particular, a naive extension of [32] by progressively convoluting the block gradient vectors and i.i.d. standard normals 
will not yield a consistent test. Specifically, note that the key to a successful bootstrap is to mimic the behavior of the estimated gradient cumulative sum (CUSUM) process

$$
\left\{\sum_{i=1}^{j} \psi\left(\hat{e}_{i}\right) \mathbf{x}_{i} / \sqrt{n}\right\}_{j=1}^{n} \quad \text { where } \hat{e}_{i}=y_{i}-\mathbf{x}_{i}^{\prime} \hat{\beta}_{n}
$$

are the residuals of the M-estimation. The bootstrap in [32] mimics the latter process by $\left\{\sum_{i=1}^{j}\left[\sum_{k=i}^{i+m} \psi\left(\hat{e}_{k}\right) \mathbf{x}_{k} / \sqrt{n m}\right] V_{i}\right\}_{j=1}^{n-m}$ where $m$ is a user-chosen block size and $V_{i}$ 's are standard normals that are independent of the the data. If the true errors $\left\{e_{i}\right\}$ were known and the residuals in (3) are replaced by the true errors, then it can be shown that the above bootstrap consistently mimics (3). However, due to the nonnegligible differences between the true errors and the residuals, it is shown that the limiting behaviors of (3) and the above bootstrap process differ, and hence the bootstrap is inconsistent.

In this paper, we extend and modify the procedure in [32] and propose an easyto-implement bootstrap methodology by combining an extension of the Powell's sandwich estimates [24] and a progressive convolution of the block sums of the estimated gradient vectors with i.i.d. standard normal auxiliary random variables. The bootstrap is shown to be consistent for a wide class of M-estimation under nonstationary temporal dependence and predictor-error dependence. The bootstrap procedure enjoys the asymptotic optimal property that it approaches the covariance structure of the target limiting Gaussian process no slower than the nearly optimal approximation rate of the Bahadur representation in various important cases such as the quantile regression. Meanwhile, we prove that our bootstrap can detect local alternatives with the optimal $1 / \sqrt{n}$ parametric rate. Furthermore, our simulation studies indicate that the gradient-based method has a superior finite sample power performance than regression-coefficient-based structural stability tests under time series nonstationarity.

There is a large amount of work in testing structural stability of parameters for general M-estimation and special cases such as least squares and quantile regressions. It is impossible to gather a complete list here and we shall only mention some representative works. For least squares regression, [9, 20] developed CUSUM tests with i.i.d. normal errors. Ploberger and Kramer [21] extended such tests to stationary and ergodic errors. [1] established Wald-type, LM, LR-like tests based on partial-sample GMM estimators with strong mixing assumptions. These test statistics are constructed through coefficients estimated by different portions of data. There are also a class of tests which heavily depend on the residuals of the least squares regression. For example, [6] obtained asymptotically distribution free test statistics associate with i.i.d. errors; see also [7] for tests of multiple structural changes. Recently, [15] and [27] investigated structural change detection for least squares regression when covariates and errors are nonstationary. For quantile 
regression, traditionally when dealing with stationary data, the regression coefficient CUSUM test is shown to be asymptotically pivotal [26] and the gradient CUSUM test is advocated over the regression coefficient test as it is asymptotically free of the densities of the errors [16, 26]. For general M-estimation, [25] investigated the gradient-based change point tests for stationary predictors and errors with predictor-error independence. We also refer to the recent review of [3] for more discussions and references. A testing strategy that prevails throughout most of the above mentioned papers is to pivotalize the test statistics. As a result, functionals of some well investigated processes, such as the Brownian bridge, can be used to approximate the large sample behaviors of the tests. However, due to the nonstationarity of the predictors and errors considered in this paper, it is shown that the gradient CUSUM process behaves complexly over time and it cannot be pivotalized in general. As a result, in general, the classic testing procedures based on the idea of pivotalization are not consistent for structural change tests under non-stationarity. We also refer to [32] for a detailed discussion in the case of testing structural changes in mean.

The rest of the paper is organized as follows. In Section 2, we will introduce the nonstationary time series models for the predictors and errors with multiple illustrative examples. A uniform Bahadur representation and related asymptotic results are established for general M-estimation under nonstationarity and temporal dependence. Section 3 proposes the structural change tests and the bootstrap and investigates their asymptotic Type I error and power behaviors. In Section 4, we perform Monte Carlo experiments to study the finite sample behaviors of the gradient-based test. Section 5 contains an empirical illustration using a public health time series. All technical proofs are relegated to the online Appendix.

2. M-estimation under time series nonstationarity. We first introduce some notation. Define $X_{n} \geq_{p} Y_{n}$ if $\mathbb{P}\left(X_{n} \geq Y_{n}\right) \rightarrow 1$ as $n \rightarrow \infty$. Similarly define “ $\leq p$." For a $p$-dimensional vector $\mathbf{v}=\left(v_{1}, \ldots, v_{p}\right)^{\prime}$, let $|\mathbf{v}|=\sqrt{\sum_{i=1}^{p} v_{i}^{2}}$. For an $m \times n$ matrix $A$, define $|A|=\sqrt{\operatorname{trace}\left(A A^{\prime}\right)}$. For a random variable $X$, let $\|X\|_{q}:=\left(\mathbb{E}|X|^{q}\right)^{1 / q}$ be its $\mathcal{L}_{q}$ norm. For a semipositive definite matrix $\Sigma$, let $\lambda_{1}(\Sigma)$ be its smallest eigenvalue. For a $p$-dimensional random vector $\mathbf{v}$, let $\|\mathbf{v}\|_{q}=\||\mathbf{v}|\|_{q}$. Write $\mathbf{v} \in \mathcal{L}_{q}$ if $\|\mathbf{v}\|_{q}<\infty$. For an $m \times n$ random matrix $A$, define $\|A\|_{q}=\||A|\|_{q}$. Write $\|\cdot\|:=\|\cdot\|_{2}$. Let $\mathcal{F}_{i}=\left(\ldots, \eta_{i-1}, \eta_{i}\right)$ and $\mathcal{F}_{i}^{(j)}=$ $\left(\ldots, \eta_{j-1}, \eta_{j}^{\prime}, \eta_{j+1}, \ldots, \eta_{i}\right)$ for $j \leq i$, where $\left(\left\{\eta_{i}\right\}_{i=-\infty}^{\infty},\left\{\eta_{j}^{\prime}\right\}_{j=-\infty}^{\infty}\right)$ are i.i.d. random variables. Write $\mathcal{F}_{i}^{*}$ for $\mathcal{F}_{i}^{(0)}$. For $x \in \mathbb{R}$, let $\lfloor x\rfloor=\max \{k \in \mathbb{Z}, k \leq x\}$, and $\lceil x\rceil=\min \{k \in \mathbb{Z}: k \geq x\}$. Write $N=\left\lfloor\frac{n}{\log n}\right\rfloor$ for short. Let " $\Rightarrow$ " denote the convergence in distribution. Throughout the paper, let $\chi \in(0,1)$ be a constant which may vary from case to case, and $M$ be a sufficiently large constant which may vary from line to line. Let $\mathbf{1}(\cdot)$ be the usual indicator function. Let $\psi(u ; \epsilon)=|\psi(u+\epsilon)|+|\psi(u-\epsilon)|$, where $\psi(\cdot)$ is the left derivative of $\rho(\cdot)$, the 
loss function of the corresponding M-estimator. For any function $f(x), x \in \mathbb{R}$ and an open interval $I$, we write $f(x) \in \mathcal{C}^{l}(I)$ if the $l$ th derivative of $f$ is continuous on $I$. Write $a \vee b$ for $\max \{a, b\}$, and $a \wedge b$ for $\min \{a, b\}$.

2.1. Nonstationary time series models. In order to model the complex temporal dynamics of the covariate and error processes, we introduce the following class of piecewise locally stationary (PLS) time series [32].

Definition 2.1. For $k<\infty$, we say that $\left\{e_{i}\right\}_{i=1}^{n}$ is a PLS process generated by filtrations $\mathcal{F}_{1, i}, \mathcal{F}_{2, i}, \ldots, \mathcal{F}_{k, i}$ with $r$ breaks $\left[\operatorname{PLS}\left(r, \mathcal{F}_{1, i}, \mathcal{F}_{2, i}, \ldots, \mathcal{F}_{k, i}\right)\right]$ if there exist constants $0=b_{0}<b_{1}<\cdots<b_{r}<b_{r+1}=1$ and nonlinear filters $G_{0}, G_{1}, \ldots, G_{r}$, such that

$$
e_{i}=G_{j}\left(t_{i}, \mathcal{F}_{1, i}, \ldots, \mathcal{F}_{k, i}\right), \quad \text { if } b_{j}<t_{i} \leq b_{j+1},
$$

where $t_{i}=i / n, \mathcal{F}_{l, i}=\left\{\ldots, \varepsilon_{l, 0}, \varepsilon_{l, 1}, \ldots, \varepsilon_{l, i}\right\}$ for $1 \leq l \leq k$. For each $l,\left\{\varepsilon_{l, i}\right\}_{i=-\infty}^{\infty}$ are i.i.d. r.v.'s. For $l \neq s,\left\{\varepsilon_{l, i}\right\}_{i=-\infty}^{\infty}$ and $\left\{\varepsilon_{s, i}\right\}_{i=-\infty}^{\infty}$ are independent.

In Definition 2.1, the functions $G_{0}, \ldots, G_{r}$ and the break points $b_{1}, \ldots, b_{r}$ are unknown nuisance parameters. If $G_{j}(t, \cdot)$ is a smooth function in $t$, then $e_{i}$ changes smoothly on $\left(b_{j}, b_{j+1}\right], j=0, \ldots, r$. The smooth change is interrupted at break points $b_{1}, \ldots, b_{r}$ where the time series can experience abrupt changes in its data generating mechanism. The PLS class is appropriate to describe stochastic temporal systems which experience occasional structural breaks and otherwise evolve smoothly over time. In this paper, we model both the covariate and error processes as PLS series to capture their complexly time-varying behaviors. To quantify the temporal dependence of PLS processes, we shall introduce the following dependence measure.

Definition 2.2. Consider the $\operatorname{PLS}\left(r, \mathcal{F}_{1, i}, \ldots, \mathcal{F}_{k, i}\right)$ process $\left\{e_{i}\right\}_{i=-\infty}^{\infty}$ defined in (4). Assume $\max _{1 \leq i \leq n}\left\|e_{i}\right\|_{p}<\infty$ for some $p>0$. Then we define $\Delta_{p}(G, l)$, the $l$ th dependence measure for $\left\{e_{i}\right\}_{i=-\infty}^{\infty}$ in $\mathcal{L}_{p}$ norm as

$$
\Delta_{p}(G, l):=\max _{0 \leq j \leq r} \sup _{b_{j}<t \leq b_{j+1}}\left\|G_{j}\left(t, \mathcal{F}_{1, l}, \ldots, \mathcal{F}_{k, l}\right)-G_{j}\left(t, \mathcal{F}_{1, l}^{*}, \ldots, \mathcal{F}_{k, l}^{*}\right)\right\|_{p} .
$$

Note that $\Delta_{p}(G, l)=0$ for $l<0$. If we view $e_{i}$ as the output of a physical system which is driven by innovations $\left\{\varepsilon_{s, i}\right\}_{i=-\infty}^{\infty}, s=1, \ldots, k$, then $\Delta_{p}(G, l)$ measures the contribution of the innovations $l$ steps ahead in generating the current observation of the system, via replacing them with i.i.d. copies and measuring the magnitude of change in the output of the system. The measure $\Delta_{p}(G, l)$ for a broad class of classic time series models can be calculated, for example, invertible ARMA process, $(\mathrm{G})$ arch models $[8,12]$ and threshold models [28]. We refer to [32] for more details about PLS models and their dependence measures. 
Throughout this paper, we assume that

$$
e_{i}=G_{k}\left(i / n, \mathcal{F}_{i}, \mathcal{G}_{i}\right)
$$

if $b_{k}<i / n \leq b_{k+1}$ with break points $0=b_{0}<b_{1}<\cdots<b_{r}<b_{r+1}=1$. Define $w(i)=j$ if $b_{j}<i / n \leq b_{j+1}$. We formulate the covariates as (where we fix $H_{k, 1} \equiv 1$ below for the intercept)

$$
\mathbf{x}_{i}=\mathbf{H}_{k}\left(i / n, \mathcal{F}_{i-1}, \mathcal{G}_{i}\right):=\left(H_{k, 1}\left(i / n, \mathcal{F}_{i-1}, \mathcal{G}_{i}\right), \ldots, H_{k, p}\left(i / n, \mathcal{F}_{i-1}, \mathcal{G}_{i}\right)\right)^{\prime}
$$

for $d_{k}<i / n \leq d_{k+1}$, where $d_{0}=0<d_{1}<\cdots<d_{s}<d_{s+1}=1$ are break points of $\left\{\mathbf{x}_{i}\right\}$. Here, the filtrations $\mathcal{G}_{i}$ and $\mathcal{F}_{i}$ are generated by $\left(\ldots, \eta_{i-1}, \eta_{i}\right)$ and $\left(\ldots, \varepsilon_{i-1}, \varepsilon_{i}\right)$, respectively, where $\left\{\eta_{i}\right\}_{i=-\infty}^{\infty}$ and $\left\{\varepsilon_{i}\right\}_{i=-\infty}^{\infty}$ are independent. Observe that the errors $\left\{e_{i}\right\}_{i=1}^{n}$ and the covariates $\left\{\mathbf{x}_{i}\right\}_{i=1}^{n}$ are allowed to be dependent as they are generated by common filtrations $\left\{\mathcal{G}_{i}\right\}_{i \in \mathbb{Z}}$ and $\left\{\mathcal{F}_{i}\right\}_{i \in \mathbb{Z}}$.

The above formulation of the error and covariate processes contains a wide range of state-heteroscedastic, exogenous and autoregressive linear regression models used in practice. The introduction of $\mathcal{F}_{i-1}$ and $\mathcal{F}_{i}$ in the covariates and errors is to accommodate autoregressive-type models where the covariates at time $i$ contain response information up to time $i-1$. On the other hand, we introduce filtrations $\mathcal{G}_{i}$ in the definitions of both $e_{i}$ and $\mathbf{x}_{i}$ to accommodate any extra information which could influence the covariates or errors. In particular, if the generating mechanisms of $\left\{e_{i}\right\}_{i=1}^{n}$ and $\mathbf{x}_{i}$ are functionally independent of $\mathcal{G}_{i}$ and $\mathcal{F}_{i-1}$, respectively, then we obtain a purely exogenous model where the covariates and errors are independent. Below we list two other frequently used subclasses of the above formulation.

EXAMPLE 1. Consider the following heteroscedastic error model:

$$
y_{i}=\mathbf{x}_{i}^{\prime} \beta+s\left(\mathbf{x}_{i}\right) \eta_{i}
$$

where $s(\cdot)$ is a piecewise smooth function, $\left\{\eta_{i}\right\}_{i=1}^{n}$ is $\operatorname{PLS}\left(r, \mathcal{F}_{i}\right)$ and $\left\{\mathbf{x}_{i}\right\}_{i=1}^{n}$ is $\operatorname{PLS}\left(s, \mathcal{G}_{i}\right)$. Furthermore, the filtrations $\left\{\mathcal{F}_{i}\right\}_{i \in \mathbb{Z}}$ and $\left\{\mathcal{G}_{i}\right\}_{i \in \mathbb{Z}}$ are independent. Note that $e_{i}=s\left(\mathbf{x}_{i}\right) \eta_{i}$ can be written as a PLS process generated by $\left(\mathcal{F}_{i}, \mathcal{G}_{i}\right)$. Lack of fit tests in regression quantiles of the above heteroscedastic error model with $\left\{\eta_{i}\right\}_{i=1}^{n}$ i.i.d. are investigated in [16], among others.

EXAMPLE 2. Consider the following autoregressive model:

$$
y_{i}=\mathbf{x}_{i}^{\prime} \beta+e_{i}
$$

where $\mathbf{x}_{i}=\left(y_{i-1}, \ldots, y_{i-p}\right)^{\prime}, \beta=\left(\beta_{1}, \ldots, \beta_{p}\right)^{\prime}, \sum_{j=1}^{p} \beta_{j} z^{j} \neq 1$ for all $|z| \leq 1+c$ with some constant $c>0$ and $\left\{e_{i}\right\}$ is $\operatorname{PLS}\left(r, \mathcal{F}_{i}\right)$. Note that $\mathbf{x}_{i}$ is a PLS process generated by $\mathcal{F}_{i-1}$. 
2.2. Asymptotic theory for M-estimation. Consider model (1). In this paper, we focus on robust loss functions in the sense that

$$
|\psi(x)-\psi(y)| \leq M_{1}+M_{2}|x-y|
$$

for all $x, y \in \mathbb{R}$ and some positive constants $M_{1}$ and $M_{2}$. It is easy to check that the left derivatives of the loss functions of quantile, expectile, $\mathcal{L}_{q}$ for $1<q<2$, least squares and the Huber regressions all satisfy (6).

The asymptotic behavior of the M-estimator $\hat{\beta}_{n}$ in (2) was investigated by numerous researchers. Among them, for quantile regression with i.i.d. error, [5] approximated $\sqrt{n}\left(\hat{\beta}_{n}-\beta\right)$ by linear forms and [18] showed that the remaining term of the approximation is of order $O_{\text {a.s. }}\left(n^{-1 / 4}(\log \log n)^{3 / 4}\right)$. Babu [4] obtained asymptotic results for strong mixing errors. Portnoy [23] acquired asymptotic approximations of $\sqrt{n}\left(\hat{\beta}_{n}-\beta\right)$ when the errors are " $m$-decomposable." With physical dependence measures, [30] obtained Bahadur representation for models with fixed design and stationary errors. As a first contribution of this paper, we obtain a Bahadur representation with nearly optimal rate (except a multiplicative logarithm factor) for model (1) with a wide class of PLS errors and regressors. The imposed conditions are mild and can be checked easily; see Proposition 2.1 and below.

For $q \geq 0, j=0,1, \ldots, r$, define for $t \in\left(b_{j}, b_{j+1}\right]$,

$$
\begin{aligned}
\Xi_{j}^{(q)}\left(t, x \mid \mathcal{F}_{k-1}, \mathcal{G}_{k}\right) & =\frac{\partial^{q}}{\partial x^{q}} \mathbb{E}\left\{\psi\left(G_{j}\left(t, \mathcal{F}_{k}, \mathcal{G}_{k}\right)+x\right) \mid \mathcal{F}_{k-1}, \mathcal{G}_{k}\right\}, \\
\bar{\kappa}_{j}\left(t, x, \mathbf{x}_{i}\right) & =\frac{\partial}{\partial x} \mathbb{E}\left(\psi\left(G_{j}\left(t, \mathcal{F}_{i}, \mathcal{G}_{i}\right)+x\right) \mid \mathbf{x}_{i}\right), \\
F_{j}^{(q)}\left(t, x \mid \mathcal{F}_{k-1}, \mathcal{G}_{k}\right) & =\frac{\partial^{q}}{\partial x^{q}} \mathbb{E}\left\{\mathbf{1}\left(G_{j}\left(t, \mathcal{F}_{k}, \mathcal{G}_{k}\right) \leq x\right) \mid \mathcal{F}_{k-1}, \mathcal{G}_{k}\right\}, \\
F_{j}^{(q)}\left(t, x \mid \mathbf{x}_{k}\right) & =\frac{\partial^{q}}{\partial x^{q}} \mathbb{E}\left\{\mathbf{1}\left(G_{j}\left(t, \mathcal{F}_{k}, \mathcal{G}_{k}\right) \leq x\right) \mid \mathbf{x}_{k}\right\}, \\
f_{j}\left(t, x \mid \mathcal{F}_{k-1}, \mathcal{G}_{k}\right) & =F_{j}^{(1)}\left(t, x \mid \mathcal{F}_{k-1}, \mathcal{G}_{k}\right), \quad f_{j}\left(t, x \mid \mathbf{x}_{k}\right)=F_{j}^{(1)}\left(t, x \mid \mathbf{x}_{k}\right) .
\end{aligned}
$$

Note that for integer $q \geq 0, f_{j}^{(q)}\left(t, x \mid \mathcal{F}_{k-1}, \mathcal{G}_{k}\right)=F_{j}^{(q+1)}\left(t, x \mid \mathcal{F}_{k-1}, \mathcal{G}_{k}\right)$. For $\tau$ th quantile regression, $F_{j}^{(q)}\left(t, x \mid \mathcal{F}_{k-1}, \mathcal{G}_{k}\right)=\tau-\Xi_{j}^{(q)}\left(t,-x \mid \mathcal{F}_{k-1}, \mathcal{G}_{k}\right)$. Also by (5), $\bar{\kappa}_{j}\left(t, x, \mathbf{x}_{i}\right)=\mathbb{E}\left(\Xi_{j}^{(1)}\left(t, x \mid \mathcal{F}_{i-1}, \mathcal{G}_{i}\right) \mid \mathbf{x}_{i}\right)$. Omit the superscript $q$ if $q=0$. The following regularity conditions are needed for the covariate and error processes:

(S0) Assume that $\max _{0 \leq i \leq r} \sup _{b_{i}<s<t \leq b_{i+1}}\left\|\frac{G_{i}\left(t, \mathcal{F}_{0}, \mathcal{G}_{0}\right)-G_{i}\left(s, \mathcal{F}_{0}, \mathcal{G}_{0}\right)}{t-s}\right\|_{v} \leq M$ for some constant $v>1$. The dependence measure of $e_{i}$ in $\mathcal{L}_{v}$ norm, $\Delta_{v}(G, k)$, satisfies $\Delta_{v}(G, k)=O\left(\chi^{k}\right)$. Assume $v=4(p+1) \vee 20$ unless otherwise specified.

(S1) Define $\overline{\boldsymbol{\Xi}}^{(q)}\left(x \mid \mathbf{x}_{i}\right)=\frac{\partial^{q}}{\partial x^{q}} \mathbb{E}\left(\psi\left(e_{i}+x\right) \mid \mathbf{x}_{i}\right)$. We require that for all $i=$ $1,2, \ldots, n$ and any $p$-dimensional vector $g$,

$$
\begin{aligned}
\mathbb{E}\left(\psi\left(e_{i}\right) \mid \mathbf{x}_{i}\right) & =\bar{\Xi}\left(0 \mid \mathbf{x}_{i}\right)=0 \quad \text { a.s. }, \\
\mathbb{E}\left(\bar{\Xi}\left(\mathbf{x}_{i}^{\prime} \delta \mid \mathbf{x}_{i}\right) \mathbf{x}_{i}^{\prime} g\right) & =\mathbb{E}\left(\bar{\Xi}^{(1)}\left(0 \mid \mathbf{x}_{i}\right) \mathbf{x}_{i}^{\prime} \delta \mathbf{x}_{i}^{\prime} g\right)+O\left(|\delta|^{2}\right)
\end{aligned}
$$

and $\bar{\Xi}^{(1)}\left(x \mid \mathbf{x}_{i}\right)>0$ a.s. for $|x| \leq \epsilon$ for some $\epsilon>0$. 
Define

$$
\nu_{i}(\delta)=\mathbb{E}\left\{\left[\psi\left(e_{i}+\left|\mathbf{x}_{i}\right||\delta|\right)-\psi\left(e_{i}-\left|\mathbf{x}_{i}\right||\delta|\right)\right]^{2}\left|\mathbf{x}_{i}\right|^{2}\right\} .
$$

Assume that $v_{i}(t)$ is continuous at $t=0$ and that, for $0 \leq j \leq r$,

$$
\begin{gathered}
\sup _{t \in\left(b_{j}, b_{j+1}\right], x, y \in \mathbb{R}}\left|\Xi_{j}\left(t, x \mid \mathcal{F}_{-1}, \mathcal{G}_{0}\right)-\Xi_{j}\left(t, y \mid \mathcal{F}_{-1}, \mathcal{G}_{0}\right)\right| \\
\leq C_{j, 1}|x-y|+C_{j, 2}\left|x^{2}-y^{2}\right|+C_{j, 3}|x-y|^{2}
\end{gathered}
$$

where $C_{j, 1}, C_{j, 2}, C_{j, 3} \in \mathcal{L}_{4}$ are $\left(\mathcal{F}_{-1}, \mathcal{G}_{0}\right)$ measurable random variables. We also require that for $0 \leq j \leq r, \Xi_{j}^{(1)}\left(t, 0 \mid \mathcal{F}_{-1}, \mathcal{G}_{0}\right)$ is stochastically Lipschitz continuous for $t \in\left(b_{j}, b_{j+1}\right]$, that is, $\exists M<\infty$, s.t. $\forall t_{1}, t_{2} \in\left(b_{j}, b_{j+1}\right], 0 \leq j \leq r$,

$$
\left\|\Xi_{j}^{(1)}\left(t_{1}, 0 \mid \mathcal{F}_{-1}, \mathcal{G}_{0}\right)-\Xi_{j}^{(1)}\left(t_{2}, 0 \mid \mathcal{F}_{-1}, \mathcal{G}_{0}\right)\right\| \leq M\left|t_{1}-t_{2}\right|
$$

(S2) For the covariates process, assume that $\Delta_{v}(\mathbf{H}, k)=O\left(\chi^{k}\right)$ and $\max _{1 \leq i \leq n}\left\|\mathbf{x}_{i}\right\|_{5 p+10} \leq M$. For all $0 \leq k \leq s$ and all $t_{1}, t_{2} \in\left(d_{k}, d_{k+1}\right]$, assume that $\left\|\mathbf{H}_{k}\left(t_{1}, \mathcal{F}_{-1}, \mathcal{G}_{0}\right)-\mathbf{H}_{k}\left(t_{2}, \mathcal{F}_{-1}, \mathcal{G}_{0}\right)\right\|_{v} \leq M\left|t_{1}-t_{2}\right|$ for $v$ defined in (S0).

A few comments on the above regularity conditions are in order. Condition (S0) [resp., (S2)] requires that the process $e_{i}$ (resp., $\left.\mathbf{x}_{i}\right)$ to be short range dependent with exponentially decaying dependence measures. Furthermore, (S0) [resp., (S2)] requires that the data generating mechanisms of $e_{i}$ (resp., $\mathbf{x}_{i}$ ) to be smooth between adjacent break points by posting certain piecewise stochastically Lipschitz continuous constraints. The assumption that $v=4(p+1) \vee 20$ in (S0) guarantees that the process $\left\{\psi\left(e_{i}\right) \mathbf{x}_{i}\right\}_{i=1}^{n}$ is a stochastically Hölder continuous PLS process with order higher than $1 / 4$. We point out that when $\psi(\cdot)$ is bounded or light-tailed, the moment requirements in (S0) and (S2) can be significantly relaxed. Our simulation results also show that our method works well under less restrictive moment conditions. However, for simplicity of presentation, we will omit the separate discussions and use (S0) and (S2) throughout this paper. (S2) also implies that $\max _{1 \leq i \leq n}\left|\mathbf{x}_{i}\right|=O_{p}\left(n^{1 /(5 p+10)}\right)$.

Assumption (S1) is necessary for the consistency of $\hat{\beta}_{n}$. Since $\psi(\cdot)$ is monotone, by Cauchy's inequality, the dominated convergence theorem and (6), the continuity of $v_{i}(t)$ at $t=0$ is satisfied whenever $e_{i}, 1 \leq i \leq n$ have continuous distribution functions. (8) holds if $\sup _{u}\left|\psi^{(1)}(u)\right|<\infty$, thus it holds for least squares regression. For quantile regression, (8) holds if $\max _{0 \leq j \leq r} \sup _{t \in\left(b_{j}, b_{j+1}\right], x \in \mathbb{R}} \mid f_{j}(t, x \mid$ $\left.\mathcal{F}_{-1}, \mathcal{G}_{0}\right) \mid<\infty$. In general, a sufficient condition for (8) is (6) with condition (A1) below, which we show in Proposition A.3 of the Supplementary Material [29].

In addition, (9) in (S1) is required for the existence of quantity $\Lambda(s)$; see equation (20) of the paper. A sufficient condition for (9) is similarly

$$
\max _{0 \leq j \leq r} \sup _{t \in\left(b_{j}, b_{j+1}\right], x \in \mathbb{R}} \int\left\|\frac{\partial}{\partial t} f_{j}^{(1)}\left(t, x \mid \mathcal{F}_{-1}, \mathcal{G}_{0}\right)\right\||\psi(x)| d x<\infty .
$$


Finally, (7) is implied by condition (10) in (A1), (S2) and (6).

For $t \in\left(b_{j}, b_{j+1}\right], 0 \leq j \leq r, \epsilon \in \mathbb{R}$, define

$$
\begin{aligned}
v_{j}^{(q)}(t, \epsilon) & =\int(\psi(x ; \epsilon)+1)\left\|f_{j}^{(q)}\left(t, x \mid \mathcal{F}_{-1}, \mathcal{G}_{0}\right)\right\|_{4} d x, \\
w_{j}^{(q)}(t, k, \epsilon) & =\int(\psi(x ; \epsilon)+1)\left\|f_{j}^{(q)}\left(t, x \mid \mathcal{F}_{k-1}, \mathcal{G}_{k}\right)-f_{j}^{(q)}\left(t, x \mid \mathcal{F}_{k-1}^{*}, \mathcal{G}_{k}^{*}\right)\right\|_{4} d x .
\end{aligned}
$$

We need the following additional conditions for the Bahadur representations:

(A0) Let $\underline{\lambda}_{n}^{a}$ be the smallest eigenvalue of $\mathbb{E}\left\{\sum_{i=1}^{\lfloor a n\rfloor} \bar{\Xi}^{(1)}\left(0 \mid \mathbf{x}_{i}\right) \mathbf{x}_{i} \mathbf{x}_{i}^{\prime} / a\right\}$ for any $a \in(0,1]$. Assume that $\forall s \in(0,1], \quad \liminf _{n \rightarrow \infty} \underline{\lambda}_{n}^{s} / n>0$, and $\liminf _{n \rightarrow \infty, s \rightarrow 0^{+}, n s \rightarrow \infty} \underline{\lambda}_{n}^{s} / n \in(0,+\infty)$.

(A1) There exist constants $\epsilon_{0}$ and $M$ such that for $0 \leq q \leq p+1, k \in \mathbb{N}$,

$$
\begin{aligned}
& \max _{0 \leq j \leq r} \sup _{t \in\left(b_{j}, b_{j+1}\right],|\epsilon| \leq \epsilon_{0}} v_{j}^{(q)}(t, \epsilon) \leq M, \\
& \max _{0 \leq j \leq r} \sup _{t \in\left(b_{j}, b_{j+1}\right],|\epsilon| \leq \epsilon_{0}} w_{j}^{(q)}(t, k, \epsilon)=O\left(\chi^{k}\right) .
\end{aligned}
$$

Furthermore, for quantile regression where $\psi(x)=\tau-\mathbf{1}(x \leq 0)$, we assume the following condition $\left(\mathrm{A} 1^{*}\right)$ instead of $(\mathrm{A} 1)$ :

$\left(\mathrm{A} 1^{*}\right)$ There exist some constant $\epsilon_{0}$ s.t. for $0 \leq q \leq p, k \in \mathbb{N}, 0 \leq j \leq s$,

$$
\begin{aligned}
& \sup _{t \in\left(d_{j}, d_{j+1}\right],|u| \leq \epsilon_{0}} \| F_{\delta(t)}^{(q)}\left(t, \mathbf{H}_{j}\left(t, \mathcal{F}_{k-1}, \mathcal{G}_{k}\right)^{\prime} u \mid \mathcal{F}_{k-1}, \mathcal{G}_{k}\right) \\
& -F_{\delta(t)}^{(q)}\left(t, \mathbf{H}_{j}\left(t, \mathcal{F}_{k-1}^{*}, \mathcal{G}_{k}^{*}\right)^{\prime} u \mid \mathcal{F}_{k-1}^{*}, \mathcal{G}_{k}^{*}\right) \|_{4}=O\left(\chi^{k}\right),
\end{aligned}
$$

where $\delta(t)=l$ if $b_{l}<t \leq b_{l+1}, 0 \leq l \leq r$.

By definition, the right-hand sides of (11) and (12) will be exactly 0 when $k<0$. Condition (A0) guarantees the consistency of $\left\{\hat{\beta}_{j}\right\}_{j=N}^{n}$ where $\hat{\beta}_{j}$ is the M-estimation coefficient using $\left(\mathbf{x}_{1}, y_{1}\right), \ldots,\left(\mathbf{x}_{j}, y_{j}\right)$. It is actually quite mild. By condition (S1) and Weyl inequality, if there exists an $\epsilon>0$ such that

$$
\min _{0 \leq k \leq s} \inf _{t \in\left(d_{k}, d_{k+1}\right]} \lambda_{1}\left(\mathbb{E}\left\{\mathbf{H}_{k}\left(t, \mathcal{F}_{-1}, \mathcal{G}_{0}\right) \mathbf{H}_{k}^{\prime}\left(t, \mathcal{F}_{-1}, \mathcal{G}_{0}\right)\right\}\right) \geq \epsilon,
$$

then (A0) is fulfilled. In other words, we only require that the matrices $\mathbb{E}\left\{\mathbf{H}_{k}(t\right.$, $\left.\left.\mathcal{F}_{-1}, \mathcal{G}_{0}\right) \mathbf{H}_{k}^{\prime}\left(t, \mathcal{F}_{-1}, \mathcal{G}_{0}\right)\right\}, 0 \leq k \leq s, t \in\left(d_{k}, d_{k+1}\right]$ are not degenerate. For (A1) and $\left(\mathrm{A} 1^{*}\right),(10)$ requires the (differentiated) conditional densities $f_{j}^{(q)}(t, x \mid$ $\left.\mathcal{F}_{-1}, \mathcal{G}_{0}\right)$ to be sufficiently light-tailed with respect to $x$. Meanwhile, (11) and (12) are short-range-dependent conditions for the processes $\left\{F_{j}^{(q)}\left(t, x \mid \mathcal{F}_{k-1}, \mathcal{G}_{k}\right)\right\}_{k=1}^{n}$. The following four examples show that conditions (A1) and (A1*) can be verified for a wide range of nonstationary time series M-estimation. 
EXAMPLE 3 (Quantile regression for PLS linear processes). Suppose we have the following PLS linear time series:

$$
G_{k}\left(t, \mathcal{F}_{i}, \mathcal{G}_{i}\right)=\sum_{j=0}^{\infty} a_{k, j}(t) \varepsilon_{i-j} h_{k}\left(t, \mathcal{G}_{i-j}\right), \quad b_{k}<t \leq b_{k+1}, 0 \leq k \leq r
$$

where $\left\{\varepsilon_{i}\right\}_{i \in \mathbb{Z}}$ are i.i.d. mean 0 r.v.'s with $\mathbb{E}\left|\varepsilon_{0}\right|^{4 u}<\infty$ for some $u>1$. In addition, $h_{k}(t, \cdot)$ and $a_{j, k}(\cdot)$ are piecewise (stochastically) Lipschitz continuous functions. Without loss of generality, let $a_{k, 0}(t) \equiv 1$. Assume that $\sup _{x \in \mathbb{R}}\left|f_{\varepsilon}^{(l)}(x)\right| \leq M<\infty$ for $0 \leq l \leq p$ where $f_{\varepsilon}(x)$ is the density function of $\varepsilon_{0}$. Write $w=u /(u-1)$. We have the following proposition.

Proposition 2.1. Assume that (i) there exists an $\eta>0$ such that $\left|h_{k}(t, \cdot)\right| \geq$ $\eta$, (ii) for $0 \leq k \leq r, t \in\left(b_{k}, b_{k+1}\right],\left\|h_{k}\left(t, \mathcal{G}_{0}\right)\right\|_{4 w} \leq C, \| h_{k}\left(t, \mathcal{G}_{i}\right)-h_{k}(t$, $\left.\mathcal{G}_{i}^{*}\right) \|_{4 w}=O\left(\chi^{i}\right), a_{k, j}(t)=O\left(\chi^{j}\right)$ and (iii):

$$
\max _{0 \leq j \leq s} \sup _{t \in\left(d_{j}, d_{j+1}\right]}\left\|\mathbf{H}_{k}\left(t, \mathcal{F}_{-1}, \mathcal{G}_{0}\right)\right\|_{4 u}<\infty, \quad \Delta_{4}(\mathbf{H}, i)=O\left(\chi^{i}\right) .
$$

Then (A1*) holds.

As a side note, for PLS linear model (13), (S0) holds if we further assume

$$
\begin{aligned}
& \mathbb{E}\left|\varepsilon_{0}\right|^{v u}<\infty, \sum_{j=0}^{\infty}\left(\max _{0 \leq k \leq r} \sup _{t \in\left(b_{k}, b_{k+1}\right]}\left|\dot{a}_{k, j}(t)\right|\right)<\infty, \\
& \max _{0 \leq k \leq r} \sup _{t, s \in\left(b_{k}, b_{k+1}\right]}\left\|h_{k}\left(t, \mathcal{G}_{0}\right)-h_{k}\left(s, \mathcal{G}_{0}\right)\right\|_{v w} \leq M|t-s| .
\end{aligned}
$$

EXAMPLE 4 (General M-estimation for PLS linear processes). Consider general M-estimation with errors following (13). Write $\theta_{\gamma}(d u)=(1+|u|)^{\gamma} d u$ and $\Delta_{\gamma}=\int \psi^{4 / 3}(x) \theta_{-\gamma}(d x)$. Then we have the following proposition.

Proposition 2.2. Assume (i) for $0 \leq k \leq r, t \in\left(b_{k}, b_{k+1}\right], \| h_{k}\left(t, \mathcal{G}_{i}\right)-$ $h_{k}\left(t, \mathcal{G}_{i}^{*}\right) \|_{8}=O\left(\chi^{i}\right), h_{k}\left(t, \mathcal{G}_{0}\right) \geq \eta>0$ and that $a_{k, j}(t)=O\left(\chi^{j}\right)$, (ii) there exists a pair of positive numbers $v_{1}, v_{2}, v_{1}^{-1}+v_{2}^{-1}=1$, such that $\varepsilon_{0} \in \mathcal{L}_{v_{1}(3 \gamma \vee 4)}$, $\max _{0 \leq k \leq r} \sup _{b_{k}<t \leq b_{k+1}}\left\|h_{k}\left(t, \mathcal{G}_{0}\right)\right\|_{v_{2}(3 \gamma \vee 4)}<\infty$, (iii) there exists $\gamma>1$ s.t. $\Delta_{\gamma}<$ $\infty$ and (iv):

$$
\sum_{q=0}^{p+2} \int\left(f_{\varepsilon}^{(q)}(u)\right)^{4} \theta_{3 \gamma}(d u)<\infty, \quad \sum_{q=0}^{p+2} \int\left(f_{\varepsilon}^{(q)}(u) u\right)^{4} \theta_{3 \gamma}(d u)<\infty,
$$

where the second inequality of (14) can be removed if for $0 \leq j \leq r, b_{j}<t \leq$ $b_{j+1}, h_{j}\left(t, \mathcal{G}_{0}\right) \equiv 1$. Then we have that condition (A1) holds. 
EXAMPLE 5 (Quantile regression for PLS nonlinear processes). Suppose the errors are generated from the following nonlinear system:

$$
G_{k}\left(t, \mathcal{F}_{i}, \mathcal{G}_{i}\right)=R_{k}\left(t, G_{k}\left(t, \mathcal{F}_{i-1}, \mathcal{G}_{i-1}\right), \varepsilon_{i}, \eta_{i}\right)
$$

for $b_{k}<t \leq b_{k+1}, 0 \leq k \leq r$. Assume that $\max _{0 \leq k \leq r}\left\|R_{k}\left(t, x_{0}, \varepsilon_{0}, \eta_{0}\right)\right\|_{v}<\infty$ for some $x_{0}$ where $v$ is defined in (S0). Let

$$
\chi_{0}=\max _{0 \leq k \leq r} \sup _{x \neq y, t \in\left(b_{k}, b_{k+1}\right]} \frac{\left\|R_{k}\left(t, x, \varepsilon_{0}, \eta_{0}\right)-R_{k}\left(t, y, \varepsilon_{0}, \eta_{0}\right)\right\|_{v}}{|x-y|} .
$$

The above formulation offers natural extensions of many frequently used stationary nonlinear time series models, for example, (G)ARCH models [8, 12], threshold models [28] and bilinear models, into the nonstationary realm. Write $\bar{F}_{k}(t, x, s, u)=\mathbb{P}\left(R_{k}\left(t, s, \varepsilon_{i}, u\right) \leq x\right)$. For quantile regression with errors following (15), we have the following proposition.

Proposition 2.3. Assume that:

(i) $0<\chi_{0}<1$,

(ii) $\max _{0 \leq k \leq r} \sup _{t \in\left(b_{k}, b_{k+1}\right]}\left\|M\left(G_{k}\left(t, \mathcal{F}_{0}, \mathcal{G}_{0}\right)\right)\right\|_{v}<\infty$, where

$$
M(x)=\max _{0 \leq k \leq r} \sup _{t, s \in\left(b_{k}, b_{k+1}\right], t \neq s} \frac{\left\|R_{k}\left(t, x, \varepsilon_{0}, \eta_{0}\right)-R_{k}\left(s, x, \varepsilon_{0}, \eta_{0}\right)\right\|_{v}}{|t-s|},
$$

(iii) for $0 \leq q \leq p$,

$$
\max _{0 \leq k \leq r} \sup _{t \in\left(b_{k}, b_{k+1}\right], x, s \in \mathbb{R}}\left|\frac{\partial^{q}}{\partial x^{q}}\left(\frac{\partial}{\partial s}+\frac{\partial}{\partial x}\right) \bar{F}_{k}\left(t, x, s, \eta_{0}\right)\right| \leq M \quad \text { a.s. }
$$

Then (15) admits a unique solution for each integer $k \in[0, r]$ and the associated $t \in\left(b_{k}, b_{k+1}\right]$. Furthermore, assume $\Delta_{4}(\mathbf{H}, i)=O\left(\chi^{i}\right)$. Then (S0), (A1*) hold.

EXAMPLE 6 (General M-estimation for PLS nonlinear processes). Assume that for $t \in\left(b_{k}, b_{k+1}\right], 0 \leq k \leq r$, and $\varepsilon_{i}$ i.i.d. with density $f_{\varepsilon}(x)$,

$$
G_{k}\left(t, \mathcal{F}_{i}, \mathcal{G}_{i}\right)=v_{k}\left(t, G_{k}\left(t, \mathcal{F}_{i-1}, \mathcal{G}_{i-1}\right), \eta_{i}\right)+\varepsilon_{i} .
$$

Consider general M-estimation with errors satisfying (16). Recall the definition of $\Delta_{\gamma}$ and $\theta_{\gamma}(d u)$ in Example 4. We have the following proposition.

Proposition 2.4. Assume (i) $\left\|v_{k}\left(t, x, \eta_{0}\right)-v_{k}\left(t, y, \eta_{0}\right)\right\|_{6 \gamma \vee 8} \leq \chi_{0}|x-y|$ for some $0<\chi_{0}<1$ and for $t \in\left(b_{k}, b_{k+1}\right], 0 \leq k \leq r$; (ii) there exists some $x_{0}$ such that $\max _{0 \leq k \leq r} \sup _{t \in\left(b_{k}, b_{k+1}\right]}\left\|v_{k}\left(t, x_{0}, \eta_{0}\right)\right\|_{6 \gamma \vee 8}<\infty$ and (iii) $\Delta_{\gamma}<\infty$ for some $\gamma>1$, and that

$$
\sum_{q=0}^{p+2} \int\left(f_{\varepsilon}^{(q)}(u)\right)^{4} \theta_{3 \gamma}(d u)<\infty
$$


Then we have that condition (A1) holds and $\Delta_{6 \gamma \vee 8}(G, l)=O\left(\chi_{0}^{l}\right)$ when errors follow (16).

For the rest of the paper, we assume that for $1 \leq i \leq n$,

$$
v_{i}(t)=O\left(|t|^{\eta}\right) \quad \text { for some } \eta>0,
$$

as it is satisfied by all quantile, expectile, least squares, the Huber and robust $\mathcal{L}_{q}$ regressions. Without the assumption, via the same techniques used in this paper, all the results can still be established but with more factors of logarithms involved. Write $d_{1, n}=n^{-1 / 2} \log n, d_{2, n}=n^{-1 / 2}(\log n)^{2}$ for short.

LEMMA 2.1. Assume (S0)-(S2), (A0) and (A1) [or (A1*) for quantile regression]. Then we have (i) $\left|\hat{\beta}_{n}-\beta\right| \leq_{p} d_{1, n}$, (ii) $\max _{N \leq j \leq n}\left|\hat{\beta}_{j}-\beta\right| \leq_{p} d_{2, n}$.

Result (i) shows that $\hat{\beta}_{n}$ is weakly consistent. Result (ii) establishes the uniform consistency of $\hat{\beta}_{j}$ estimated by different subsamples with at least $N$ observations. The consistency results are needed for the structural stability test in Section 3 . The following theorem establishes an important uniform Bahadur representation for a wide class of nonstationary time series M-estimation.

THEOREM 2.1. Write $\Lambda(j)=\mathbb{E}\left\{\sum_{i=1}^{j} \bar{\kappa}_{w(i)}\left(i / n, 0, \mathbf{x}_{i}\right) \mathbf{x}_{i} \mathbf{x}_{i}^{\prime} / n\right\}$. Assume that $\max _{0 \leq j \leq r} \sup _{t \in\left(b_{j}, b_{j+1}\right], x \in \mathbb{R}}\left|f_{j}\left(t, x \mid \mathcal{F}_{-1}, \mathcal{G}_{0}\right)\right|<\infty$. Then under assumptions (S0)-(S2), (A0) and (A1) [or (A1*) for quantile regression], we have (i):

$$
\begin{gathered}
\sqrt{n}\left(\hat{\beta}_{n}-\beta\right)-\left(\hat{\Lambda}(n) n^{1 / 2}\right)^{-1} \sum_{i=1}^{n} \psi\left(e_{i}\right) \mathbf{x}_{i} \\
=O_{p}\left(\sqrt{\frac{\sum_{i=1}^{n} v_{i}\left(d_{1, n}\right)}{n}} \log n+r_{n}\right),
\end{gathered}
$$

and (ii):

$$
\begin{gathered}
\max _{N \leq j \leq n}\left|\sqrt{n}\left(\hat{\beta}_{j}-\beta\right)-\left(\hat{\Lambda}(j) n^{1 / 2}\right)^{-1} \sum_{i=1}^{j} \psi\left(e_{i}\right) \mathbf{x}_{i}\right| \\
=O_{p}\left(\sqrt{\frac{\sum_{i=1}^{n} v_{i}\left(d_{2, n}\right)}{n}} \log n+r_{n}\right) .
\end{gathered}
$$

The quantity $r_{n}$ equals zero if $\psi(\cdot)$ is continuous, otherwise $r_{n}=n^{\frac{1}{5 p+10}-1 / 2}$.

REMARK 2.1 [The order of the quantity $v_{i}(\delta)$ ]. For quantile regression, if for $i=1, \ldots, n$, the conditional densities satisfy

$$
\max _{0 \leq k \leq r} \sup _{t \in\left(b_{k}, b_{k+1}\right], x \in \mathbb{R}}\left|f_{k}\left(t, x \mid \mathbf{x}_{i}\right)\right| \leq M_{0}<\infty \quad \text { a.s., }
$$


then we have $v_{i}(\delta)=O(|\delta|)$, which results in that $\sqrt{\sum_{i=1}^{n} v_{i}\left(d_{2, n}\right)}=O\left(n^{1 / 4} \times\right.$ $\left.\log ^{2} n\right)$. As in the discussion of Example 1 of [30], for the Huber, $\mathcal{L}_{2}$ and expectile regressions, there exists $u$ such that for $1 \leq i \leq n, \sup _{|\delta| \leq u}\left\|\psi^{\prime}\left(e_{i}+\left|\mathbf{x}_{i} \| \delta\right|\right)\right\|_{4} \leq$ $M<\infty$. Hence $v_{i}(\delta)=O\left(\delta^{2}\right)$, and consequently $\sqrt{\sum_{i=1}^{n} v_{i}\left(d_{2, n}\right)}=O\left(\log ^{2} n\right)$. For robust $\mathcal{L}_{q}$ regression, noting that $v_{i}(t) \leq 2\left(v_{i}^{+}(t)+v_{i}^{-}(t)\right)$, where

$$
\begin{aligned}
& v_{i}^{+}(\delta)=\mathbb{E}\left\{\left[\psi\left(e_{i}+\left|\mathbf{x}_{i}\right||\delta|\right)-\psi\left(e_{i}\right)\right]^{2}\left|\mathbf{x}_{i}\right|^{2}\right\}, \\
& v_{i}^{-}(\delta)=\mathbb{E}\left\{\left[\psi\left(e_{i}\right)-\psi\left(e_{i}-\left|\mathbf{x}_{i}\right||\delta|\right)\right]^{2}\left|\mathbf{x}_{i}\right|^{2}\right\} .
\end{aligned}
$$

By Lemma 4 of [2], we have that for $3 / 2<q<2$,

$$
v_{i}^{+}(\delta) \leq 36 \delta^{2} \mathbb{E}\left(\left|\mathbf{x}_{i}\right|^{4}\left|e_{i}\right|^{2 q-4}\right) .
$$

Since $2 q-4>-1$, assuming (19), we get

$$
\begin{aligned}
\mathbb{E}\left(\left|e_{i}\right|^{2 q-4} \mid \mathbf{x}_{i}\right) & =\mathbb{E}\left(\int|y|^{2 q-4} f_{w(i)}\left(i / n, y \mid \mathbf{x}_{i}\right) d y\right) \\
& \leq \mathbb{E}\left(M_{0} \int_{0}^{1}|y|^{2 q-4} d y+\int_{1}^{\infty} f_{w(i)}\left(i / n, y \mid \mathbf{x}_{i}\right) d y\right) \leq M .
\end{aligned}
$$

By applying similar arguments to $v_{i}^{-}(\delta)$, we have that $v_{i}(\delta)=O\left(\delta^{2}\right)$. For $1<q \leq$ $3 / 2$, by Lemma 4 of [2], we obtain

$$
v_{i}^{+}(\delta) \leq 36 \delta^{2} \mathbb{E}\left(\left|\mathbf{x}_{i}\right|^{4}\left|e_{i}\right|^{2 q-4} \mathbf{1}\left(\left|e_{i}\right| \geq \delta\right)\right)+2^{4-2 q}|\delta|^{2 q-2} \mathbb{E}\left(\left|\mathbf{x}_{i}\right|^{2 q} \mathbf{1}\left(\left|e_{i}\right| \leq \delta\right)\right) .
$$

For $q=3 / 2$, by (19), we have $\mathbb{E}\left(\left|e_{i}\right|^{2 q-4} \mathbf{1}\left(\left|e_{i}\right| \geq \delta\right) \mid \mathbf{x}_{i}\right) \leq M \delta^{2 q-3} \log \left(|\delta|^{-1}\right)$ and $\mathbb{E}\left(\mathbf{1}\left(\left|e_{i}\right| \leq \delta\right) \mid \mathbf{x}_{i}\right) \leq M \delta$, which lead to that $v_{i}^{+}(\delta)=O\left(|\delta|^{2 q-1} \log \left(|\delta|^{-1}\right)\right)$. By applying similar arguments to $v_{i}^{-}(\delta)$, we have $v_{i}(\delta)=O\left(|\delta|^{2 q-1} \log \left(|\delta|^{-1}\right)\right)$. Similar but easier arguments show that $v_{i}(\delta)=O\left(|\delta|^{2 q-1}\right)$ for $1<q<3 / 2$.

In Theorem 2.1(i) establishes a Bahadur representation of $\hat{\beta}_{n}$ for nonstationary time series M-estimation and (ii) establishes a uniform Bahadur representation of $\left\{\hat{\beta}_{j}, N \leq j \leq n\right\}$. When $\mathcal{L}_{1}$ loss is applied, both results almost achieve the optimal order $n^{-1 / 4}(\log \log n)^{3 / 4}$ except a factor of multiplicative logarithms. For Huber, $\mathcal{L}_{q}, 3 / 2<q \leq 2$ and expectile regressions, according to Remark 2.1, the approximation rates are $\log ^{2} n$ and $\log ^{3} n$ in (i) and (ii), respectively. The latter rates are again nearly optimal except a factor of multiplicative logarithms. Observe that, due to the nonstationarity, the approximating processes depend on $\left\{\bar{\kappa}_{w(i)}\left(i / n, 0, \mathbf{x}_{i}\right), 1 \leq i \leq n\right\}$, which are the conditional densities of the errors $e_{i}$ in the scenario of quantile regression. [23] also provided a similar form of Bahadur representation with nonstationary errors for quantile regression.

3. Structural stability tests. We are now ready to propose and investigate change point tests for general nonstationary time series M-estimation. 
3.1. Test statistics. Consider a general nonparametric M-estimation model of the form:

$$
y_{i}=\mathbf{x}_{i}^{\prime} \beta_{i}+e_{i}, \quad i=1,2, \ldots, n .
$$

We are interested in testing whether $\beta_{i}$ 's remain constant over time. That is, we test

$$
H_{0}: \beta_{1}=\beta_{2}=\cdots=\beta_{n}=\beta \leftrightarrow H_{A}: \beta_{i} \neq \beta_{j} \quad \text { for some } 1 \leq i<j \leq n
$$

for some unknown $\beta$. Consider the following test statistic:

$$
T_{n}=\max _{1 \leq j \leq n}\left|\frac{\sum_{i=1}^{j} \psi\left(\hat{e}_{i, n}\right) \mathbf{x}_{i}}{\sqrt{n}}\right|,
$$

where $\hat{e}_{i, n}=y_{i}-\mathbf{x}_{i}^{\prime} \hat{\beta}_{n}$ are the residuals. The test statistic $T_{n}$ is the CUSUM statistic of the estimated gradient vectors of the regression. If $H_{0}$ is violated, then $T_{n}$ tends to be large. In the following, we shall investigate the asymptotic null distribution and power behavior of $T_{n}$ in detail. Note that, under conditions (S0)-(S2), $\psi\left(e_{i}\right) \mathbf{x}_{i}$ can be viewed as a realization from a PLS process with $r_{1}$ break points $c_{1}, \ldots, c_{r_{1}}$, namely, $\tilde{G}_{v(i)}\left(t, \mathcal{F}_{i}, \mathcal{G}_{i}\right)$, where $v(i)=k$ for $c_{k}<i / n \leq c_{k+1}$. We set $c_{0}=0$ and $c_{r_{1}+1}=1$. Then $\psi\left(e_{i}\right) \mathbf{x}_{i}=\tilde{G}_{v(i)}\left(i / n, \mathcal{F}_{i}, \mathcal{G}_{i}\right)$. Here, $r_{1}=|A \cup B|$, where $A=$ $\left\{b_{1}, \ldots, b_{r}\right\}$ is the set of break points of the errors, and $B=\left\{d_{1}, \ldots, d_{s}\right\}$ is the set of break points of the covariates. Here, $|\cdot|$ denotes the cardinality of a set. The detailed mathematical form of $\tilde{G}_{v(i)}\left(t, \mathcal{F}_{i}, \mathcal{G}_{i}\right)$ is complex which we treat as a nuisance parameter. Define the long-run covariance matrices:

$$
\Sigma^{2}(t)=\sum_{h=-\infty}^{\infty} \operatorname{Cov}\left(\tilde{G}_{k}\left(t, \mathcal{F}_{0}, \mathcal{G}_{0}\right), \tilde{G}_{k}\left(t, \mathcal{F}_{h}, \mathcal{G}_{h}\right)\right), \quad t \in\left(c_{k}, c_{k+1}\right], 0 \leq k \leq r_{1}
$$

Let $\Sigma^{2}(0)=\lim _{t \downarrow 0} \Sigma^{2}(t)$. In order to investigate the limiting behavior of $T_{n}$, we shall further introduce the following assumption:

(A2) The smallest eigenvalue of $\Sigma^{2}(t)$ is bounded away from 0 for $t \in[0,1]$.

It is shown in Proposition A.2 in the Supplementary Material [29] that the dependence of $\left\{\psi\left(e_{i}\right) \mathbf{x}_{i}\right\}_{i=1}^{n}$ decays exponentially fast to 0 . Meanwhile, condition (A2) assures that the long run variance of $\psi\left(e_{i}\right) \mathbf{x}_{i}$ is not degenerate over time, which is a mild requirement. We have the following proposition, which is useful in the asymptotic study of the process $\left\{\psi\left(e_{i}\right) \mathbf{x}_{i}\right\}_{i=1}^{n}$.

Proposition 3.1. Let $f_{k}(t, x)$ be the density of $G_{k}\left(t, \mathcal{F}_{0}, \mathcal{G}_{0}\right)$. Assume (A2), (S0)-(S2) with $v=4(p+1)$. Assume that (i) there exists a sufficiently small positive เ such that

$$
\sup _{0 \leq k \leq r, t \in\left(b_{k}, b_{k+1}\right]}\left\|\psi\left(G_{k}\left(t, \mathcal{F}_{0}, \mathcal{G}_{0}\right)\right)\right\|_{\frac{4(p+1)}{p}+\iota} \leq M<\infty
$$


and (ii) $\int \psi(x ; 1)\left|\frac{\partial}{\partial x} f_{k}(t, x)\right| d x$ is finite for $0 \leq k \leq r, t \in\left(b_{k}, b_{k+1}\right]$. Then on $a$ possibly richer probability space, there exists a p-dimensional zero-mean Gaussian process $U(t)$ with covariance function $\gamma(t, s)=\int_{0}^{\min (t, s)} \Sigma^{2}(r) d r$, such that

$$
\max _{1 \leq j \leq n}\left|\frac{1}{\sqrt{n}} \sum_{i=1}^{j} \psi\left(e_{i}\right) \mathbf{x}_{i}-U(j / n)\right|=o_{p}\left(n^{-1 / 4} \log ^{2} n\right) .
$$

Write $\Lambda(t)=\lim _{n \rightarrow \infty} \mathbb{E}\left\{\sum_{i=1}^{\lfloor n t\rfloor} \bar{\kappa}_{w(i)}\left(i / n, 0, \mathbf{x}_{i}\right) \mathbf{x}_{i} \mathbf{x}_{i}^{\prime}\right\} / n$. Since $\mathbf{x}_{i}$ is $\left(\mathcal{F}_{i-1}, \mathcal{G}_{i}\right)$ measurable, we have that

$$
\mathbb{E}\left\{\sum_{i=1}^{\lfloor n t\rfloor} \bar{\kappa}_{w(i)}\left(i / n, 0, \mathbf{x}_{i}\right) \mathbf{x}_{i} \mathbf{x}_{i}^{\prime}\right\}=\mathbb{E}\left\{\sum_{i=1}^{\lfloor n t\rfloor} \Xi_{w(i)}^{(1)}\left(i / n, 0 \mid \mathcal{F}_{i-1}, \mathcal{G}_{i}\right) \mathbf{x}_{i} \mathbf{x}_{i}^{\prime}\right\} .
$$

Without loss of generality, suppose that the covariates and the errors have the same break points, that is, $\left\{b_{1}, \ldots, b_{r}\right\}=\left\{d_{1}, \ldots, d_{s}\right\}$. Then by (9) in (S1), we have that for $s \in\left(b_{j}, b_{j+1}\right]$,

$$
\begin{aligned}
\Lambda(s)= & \sum_{l=0}^{j-1} \int_{b_{l}}^{b_{l+1}} \mathbb{E}\left\{\Xi_{l}^{(1)}\left(t, 0 \mid \mathcal{F}_{-1}, \mathcal{G}_{0}\right) \mathbf{H}_{l}\left(t, \mathcal{F}_{-1}, \mathcal{G}_{0}\right) \mathbf{H}_{l}\left(t, \mathcal{F}_{-1}, \mathcal{G}_{0}\right)^{\prime}\right\} d t \\
& +\int_{b_{j}}^{s} \mathbb{E}\left\{\Xi_{j}^{(1)}\left(t, 0 \mid \mathcal{F}_{-1}, \mathcal{G}_{0}\right) \mathbf{H}_{j}\left(t, \mathcal{F}_{-1}, \mathcal{G}_{0}\right) \mathbf{H}_{j}\left(t, \mathcal{F}_{-1}, \mathcal{G}_{0}\right)^{\prime}\right\} d t
\end{aligned}
$$

The following theorem establishes the limiting null distribution of $T_{n}$ for nonstationary time series M-estimation.

THEOREM 3.1. Assume that $\max _{0 \leq j \leq r} \sup _{t \in\left(b_{j}, b_{j+1},\right] x \in \mathbb{R}}\left|f_{j}\left(t, x \mid \mathcal{F}_{-1}, \mathcal{G}_{0}\right)\right|<$ $\infty$. Suppose (S0)-(S2), (A0)-(A2) and the conditions of Proposition 3.1 hold. Then under the null hypothesis of no structural change, we have

$$
T_{n} \Rightarrow \sup _{t \in(0,1]}|G(t)|:=\sup _{t \in(0,1]}\left|U(t)-\Lambda(t) \Lambda^{-1}(1) U(1)\right|,
$$

where $U(t)$ is defined in Proposition 3.1, and $\Lambda(t)$ is defined in (20).

Theorem 3.1 establishes that $T_{n}$ converges to the maximum of certain centered Gaussian process. Two important observations should be made. First, the Gaussian process $U(t)$ is not pivotal and it has a complex covariance structure $\gamma(t, s)=$ $\int_{0}^{\min (t, s)} \Sigma^{2}(r) d r$. In particular, $\Sigma^{2}(s)$ can change both smoothly and abruptly on $[0,1]$, and hence it is inappropriate to perform $T_{n}$ by checking quantile tables of certain pivotal Gaussian processes (such as the Brownian bridge). Second, due to the nonstationarity, $\Lambda(t) \Lambda^{-1}(1)$ no longer equals $t I_{p}$ as in the stationary case, where $I_{p}$ is the $p \times p$ identity matrix. In particular, the gradient CUSUM test $T_{n}$ is no longer asymptotically free of the density functions of $\left\{e_{i}\right\}_{i=1}^{n}$ and the 
ratio $\Lambda(t) \Lambda^{-1}(1)$ should be estimated when performing the gradient CUSUM test for nonstationary time series M-estimation. Consequently, the independent wild bootstrap procedure in [16] will in general yield inconsistent testing results under nonstationarity.

The following theorem studies the asymptotic power behavior of the test for nonstationary time series M-estimation. For any bounded piecewise Lipschitz continuous $p \times 1$ vector function $g(\cdot)$, write

$$
\Lambda(s, g(\cdot))=\lim _{n \rightarrow \infty} \mathbb{E}\left\{\sum_{i=1}^{\lfloor n t\rfloor} \bar{\kappa}_{w(i)}\left(i / n, 0, \mathbf{x}_{i}\right) \mathbf{x}_{i} \mathbf{x}_{i}^{\prime} g(i / n) / n\right\} .
$$

By the arguments of $(20), \Lambda(s, g(\cdot))$ is well defined. Write

$$
\digamma(t, g(\cdot))=\Lambda(t, g(\cdot))-\Lambda(t) \Lambda(1)^{-1} \Lambda(1, g(\cdot)) .
$$

THEOREM 3.2. Consider the alternative model $H_{A}: \beta_{i}=\beta+L_{n} g(i / n)$, where $g(\cdot)$ is a bounded nonconstant piecewise Lipschitz continuous $p \times 1$ vector function defined in $[0,1]$. Suppose that (S0)-(S2), (A0)-(A2) and the conditions of Proposition 3.1 hold. Assume

$$
\max _{0 \leq k \leq r} \sup _{t \in\left(b_{k}, b_{k+1}\right], x \in \mathbb{R}}\left|f_{k}^{(j)}\left(t, x \mid \mathcal{F}_{-1}, \mathcal{G}_{0}\right)\right|<\infty
$$

for $0 \leq j \leq 3$. For quantile regression, assumes (A1*) instead of (A1). Then we have:

(i) if $L_{n}=n^{-1 / 2}$,

$$
T_{n} \Rightarrow \sup _{0<t \leq 1}|G(t)+\digamma(t, g(\cdot))|,
$$

where $G(t)$ is defined in Theorem 3.1.

(ii) If the deterministic sequence $L_{n}$ satisfies $L_{n}=o(1), \frac{\sqrt{\sum_{i=1}^{n} \nu_{i}\left(L_{n}\right)}}{\sqrt{n}} \log n \rightarrow$ $0, \sqrt{n} L_{n} \rightarrow \infty$, then $T_{n} \rightarrow{ }_{p} \infty$ at the rate $\sqrt{n} L_{n}$.

Theorem 3.2 shows that the power of the test converges to 1 if $\sqrt{n} L_{n} \rightarrow \infty$, $L_{n}=o(1)$ and $\frac{\sqrt{\sum_{i=1}^{n} v_{i}\left(L_{n}\right)}}{\sqrt{n}} \log n \rightarrow 0$, which implies that our test can detect local alternatives at the same rate $n^{-1 / 2}$ as the classic stationary case.

3.2. The bootstrap. Theorem 3.1 reveals that the key to accurate tests under nonstationarity is to consistently mimic the behaviors of the processes $\{\Lambda(t)\}$ and $\{U(t)\}$. A straightforward way to generate the limiting distribution in Theorem 3.1 is to directly estimate $\{\Lambda(t)\}$ and $\{U(t)\}$, which involves the estimation of conditional densities and long-run covariances $\Sigma^{2}(t)$ over time $t$, respectively. However, this approach is not operational in practice for the following two reasons. 
First, the estimation of the densities and the long-run covariance at a fixed time $t$ requires a total of four bandwidth parameters, which are difficult to choose in practice and can cause inaccurate testing results for moderate samples. Second, the nonparametric estimates of $\{\Lambda(t)\}$ and $\Sigma^{2}(t)$ are inconsistent near the break points of the PLS errors and covariates. Hence, it is unclear whether those plugin procedures asymptotically achieve the nominal size. In this section, we shall propose a bootstrap procedure, which avoids directly estimating the densities and long-run covariances while requiring only two tuning parameters. The proposed bootstrap procedure combines the advantages of moving block bootstrap [19] and subsampling [22] by progressively convoluting block partial sums of the estimated gradient vectors and auxiliary standard normals in order to preserve the temporal dependence structure and to mimic the pattern of the nonstationarity over time. Furthermore, in our bootstrap, we make use of an extension of the "Powell Sandwich" [24] to optimally estimate $\{\Lambda(t)\}$. In the following, we shall discuss the approximations of $\{\Lambda(t)\}$ and $\{U(t)\}$ separately.

Let $c_{n}$ be a bandwidth parameter. Define $\hat{\Lambda}_{c_{n}}(t)=\hat{\lambda}_{c_{n}}(\lfloor n t\rfloor)$, where

$$
\hat{\lambda}_{c_{n}}(j)=\sum_{i=1}^{j} \frac{\left(\psi\left(\hat{e}_{i, n}+c_{n}\right)-\psi\left(\hat{e}_{i, n}-c_{n}\right)\right) \mathbf{x}_{i} \mathbf{x}_{i}^{\prime}}{2 n c_{n}} .
$$

Note that for least squares regression, $\hat{\lambda}_{c_{n}}(j)$ in (22) equals $\sum_{i=1}^{j} \mathbf{x}_{i} \mathbf{x}_{i}^{\prime} / n$ which is independent of $c_{n}$. In addition, for quantile regression, we propose another smooth estimator for $\{\Lambda(t)\}_{t \in(0,1]}$. Define

$$
\hat{\lambda}_{c_{n}}(j)=\sum_{i=1}^{j} \frac{K\left(\hat{e}_{i, n} / c_{n}\right) \mathbf{x}_{i} \mathbf{x}_{i}^{\prime}}{n c_{n}}
$$

where $K(\cdot)$ is a symmetric smooth kernel function with bounded second order derivative, satisfying $\int K(x) d x=1, \int K(x) x^{2} d x \leq M, \int K^{2}(x) d x \leq M$ and $\int K^{\prime 2}(x) d x \leq M$. The following theorem states that $\left\{\hat{\Lambda}_{c_{n}}(t)\right\}_{t \in(0,1]}$ could be used to approximate $\{\Lambda(t)\}_{t \in(0,1]}$ uniformly.

THEOREM 3.3. Assume (S0)-(S2), (A0) and (A1) with $0 \leq q \leq(3 \vee$ $(p+1))\left[\right.$ or $\left(\mathrm{A} 1^{*}\right)$ for quantile regression]. Further assume for $0 \leq s \leq 3$, $\max _{0 \leq k \leq r} \sup _{b_{k}<t \leq b_{k+1}, x \in \mathbb{R}}\left|f_{k}^{(s)}\left(t, x \mid \mathcal{F}_{-1}, \mathcal{G}_{0}\right)\right|<\infty$. Then (i) assuming $c_{n} \rightarrow 0$, $\frac{\sqrt{\sum_{i=1}^{n} v_{i}\left(c_{n}\right) \log n}}{n c_{n}} \rightarrow 0, n c_{n}^{2} / \log ^{2} n \rightarrow \infty$, we have

$$
\sup _{t \in(0,1]}\left|\hat{\Lambda}_{c_{n}}(t)-\Lambda(t)\right|=O_{p}\left(\frac{\left(\sqrt{\sum_{i=1}^{n} v_{i}\left(c_{n}\right)} \vee 1\right) \log n}{n c_{n}}+c_{n}^{2}+n^{-1 / 2} \log n\right) .
$$

(ii) If smooth estimator (23) is used for quantile regression, assuming $\max _{0 \leq j \leq r} \sup _{b_{j}<t \leq b_{j+1}, x \in \mathbb{R}}\left\|f_{j}^{(q)}\left(t, x \mid \mathcal{F}_{i-1}, \mathcal{G}_{i}\right)-f_{j}^{(q)}\left(t, x \mid \mathcal{F}_{i-1}^{*}, \mathcal{G}_{i}^{*}\right)\right\|=O\left(\chi^{i}\right)$ 
for $q=0,1, c_{n} \log ^{2} n \rightarrow 0$ and $n c_{n}^{3} / \log ^{2} n \rightarrow \infty$, then we have

$$
\sup _{t \in(0,1]}\left|\hat{\Lambda}_{c_{n}}(t)-\Lambda(t)\right|=O_{p}\left(n^{-1 / 2} c_{n}^{-1 / 2}+c_{n}^{2}+\frac{\log ^{2} n}{n c_{n}^{3}}\right) \text {. }
$$

In fact, $\hat{\lambda}_{c_{n}}(j)$ is an extension of the "Powell's Sandwich." Furthermore, for quantile regression, $\hat{\lambda}_{c_{n}}(j)$ can be viewed as a progressive local constant kernel estimation of integrated conditional densities. Theorem 3.3 shows that $\left\{\hat{\Lambda}_{c_{n}}(t)\right\}_{t \in(0,1]}$ are uniformly consistent estimators of $\{\Lambda(t)\}_{t \in(0,1]}$. Elementary calculations show that, even with PLS errors, the optimal bandwidth $c_{n}$ for Theorem 3.3 is almost of the order of $n^{-1 / 5}$ for quantile regression. Therefore, the convergence rate of Theorem 3.3 is still almost at the order of $n^{-2 / 5}$ except a factor of multiplicative logarithms, where the order $n^{-2 / 5}$ is the well-known optimal approximation rate of the Powell's sandwich estimates for i.i.d. data. Note that the nearly $n^{-2 / 5}$ rate above is faster than $n^{-1 / 4} \log ^{2} n$, which is the nearly optimal approximation rate of the Bahadur representation in (18). For the Huber, expectile and $\mathcal{L}_{q}, q \in(3 / 2,2]$ regressions, our method also achieves the almost optimal rate $n^{-1 / 2} \log n$ when $c_{n}$ satisfies the stated bandwidth conditions and converges to zero no slower than $n^{-1 / 4}$ and no faster than $n^{-1 / 2}$. For $\mathcal{L}_{1.5}$, the convergence rate could achieve $n^{-1 / 2} \log ^{2} n$.

The remaining task for evaluating the critical values is to find a simple and datadriven way to simulate the nonstationary Gaussian process $U(t)$. The covariance structure of $U(t)$ could be quite complex. In particular, it does not necessarily have stationary increments. We propose the following gradient-based process $\tilde{\Psi}_{m, n}(t)$ to bootstrap $U(t)$ :

$$
\begin{aligned}
\tilde{\Psi}_{m, n}(t)= & \Psi_{\lfloor n t\rfloor, m}+(n t-\lfloor n t\rfloor)\left(\Psi_{\lfloor n t\rfloor+1, m}-\Psi_{\lfloor n t\rfloor, m}\right), \\
\Psi_{i, m}= & \sum_{j=1}^{i} \frac{1}{\sqrt{m(n-m+1)}} \\
& \times\left(\hat{\varpi}_{j, m}-\frac{m}{n} \hat{\varpi}_{n}\right) R_{j}, \quad i=1, \ldots, n-m+1,
\end{aligned}
$$

where $\hat{\varpi}_{j, m}=\sum_{r=j}^{j+m-1} \psi\left(\hat{e}_{r, n}\right) \mathbf{x}_{r}, \hat{\varpi}_{n}=\hat{\varpi}_{1, n}$ and $\left(R_{i}\right)_{i=1}^{n}$ are i.i.d. standard normals independent of $\left\{\mathcal{F}_{i}\right\}_{i=-\infty}^{\infty},\left\{\mathcal{G}_{i}\right\}_{i=-\infty}^{\infty}$. The consistency of $\left\{\tilde{\Psi}_{m, n}(t)\right\}$ as an estimate of $\{U(t)\}$ is provided by the following theorem.

THEOREM 3.4. Assume that $\max _{0 \leq j \leq r} \sup _{t \in\left(b_{j}, b_{j+1}\right], x \in \mathbb{R}}\left|f_{j}\left(t, x \mid \mathcal{F}_{-1}, \mathcal{G}_{0}\right)\right|<$ $\infty$ and the bandwidth $m=m(n)$ satisfies $m \rightarrow \infty, m / n \rightarrow 0$. Suppose (S0)(S2), (A0)-(A2) and the conditions of Proposition 3.1 hold. Further assume that there exists some constant $\varepsilon_{0}$, such that for $m$ and $1 \leq j \leq n-m+1$, $\left|\sum_{r=j}^{j+m-1} v_{r}(\delta)\right| \leq M \frac{m}{n} \sum_{i=1}^{n} v_{i}(\delta)$ for all $|\delta| \leq \varepsilon_{0}$. Then conditional on $\left(\mathcal{F}_{n}, \mathcal{G}_{n}\right)$, $\tilde{\Psi}_{m, n}(t) \Rightarrow U(t)$ on $\mathcal{C}(0,1)$ with the uniform topology. 
By the proof of Theorem 3.4, for quantile regressions, if we further require $\sqrt{n} \log n / m \rightarrow \infty$, then conditional on $\left(\mathcal{F}_{n}, \mathcal{G}_{n}\right)$, the covariance function of $\tilde{\Psi}_{m, n}(t)$ converges uniformly to that of $U(t)$ at the rate $n^{-1 / 4} \log ^{3 / 2} n$, which is also of the same order of the nearly optimal approximation rate of the Bahadur representation in (17). Therefore, Theorem 3.3 and Theorem 3.4 suggest that our bootstrap approaches the covariance structure of the target limiting Gaussian process no slower than the nearly optimal approximation rate of the Bahadur representation. We also note that for the Huber, $\mathcal{L}_{q}, 3 / 2 \leq q \leq 2$ and expectile regressions, by Remark 2.1, the optimal Bahadur representation rate is almost of the order of $\frac{1}{\sqrt{n}}$ except a fact of multiplicative of logarithms, which could not be archived by Theorem 3.4. The reason is that the optimal approximation rate of the robust bootstrap is of the order $n^{-1 / 3}$; see [32].

In Theorem 3.2, we show that under $H_{A}$, if $L_{n} \rightarrow 0, \frac{\sqrt{\sum_{i=1}^{n} v_{i}\left(L_{n}\right)}}{\sqrt{n}} \log n \rightarrow 0$ and $\sqrt{n} L_{n} \rightarrow \infty$, the test statistic goes to infinity at the rate $\sqrt{n} L_{n}$. Proposition B.1 in the Supplementary Material [29] further discusses the property of $\left\{\hat{\Lambda}_{c_{n}}(t)\right\}_{t \in(0,1]}$ and $\tilde{\Psi}_{m, n}(t)$ under the local alternative hypotheses. Assumes that $\frac{m \log ^{8} n}{n}=o(1)$. Then the divergence rate of $T_{n}$ under the local alternatives in (ii) of Theorem 3.2 is $\sqrt{n} L_{n}$, which is faster than $\sqrt{m}\left(L_{n} \vee \frac{\log ^{2} n}{\sqrt{n}}\right) \log n$, the fastest possible rate at which $\tilde{\Psi}_{m, n}(t)$ can go to infinity by Proposition B.1. Hence, Theorem 3.2 together with Proposition B.1 shows that our bootstrap method has asymptotic power 1 under the considered local alternatives in (ii) of Theorem 3.2. In particular, our bootstrap can detect local alternatives with the optimal $n^{-1 / 2}$ parametric rate.

REMARK 3.1. The limiting distribution of the test statistic, and hence the nonlocal power, is hard to evaluate when $L_{n}=1$ due to the time series nonstationarity and the possibly nondifferentiable gradient function. When $L_{n} \gg 1$, [17] proposes to replace $\hat{e}_{i}$ with $\tilde{e}_{i}$ to deal with the nonlocal power issue, where $\hat{e}_{i}$ is the residual of the parametric linear regression under the null hypothesis, and $\tilde{e}_{i}$ is the residual of a general nonparametric regression. We only consider very general form of alternatives. Hence, [17] is not directly applicable. We leave the problem of nonlocal power as a rewarding future work.

Combining Theorems 3.3 and 3.4, we have the following step-by-step implementation procedures for performing structural change tests for nonstationary time series M-estimation.

AlgORITHM 3.1. (i) By Section 3.3, select appropriate $m$ and $c_{n}$.

(ii) Apply Theorem 3.3 to get $\hat{\lambda}_{c_{n}}(j), j=1, \ldots, n$. Use Theorem 3.4 to generate B (say 2000) conditional i.i.d. copies $\left\{\Psi_{i, m}^{(r)}\right\}_{i=1}^{n-m+1}, r=1, \ldots, B$.

(iii) Calculate $F_{i}^{(r)}=\Psi_{i, m}^{(r)}-\hat{\lambda}_{c_{n}}(i) \hat{\lambda}_{c_{n}}^{-1}(n-m+1) \Psi_{n-m+1, m}^{(r)}$ for $r=1, \ldots, B$, $i=m, \ldots, n-m+1$. 
(iv) Let $F_{r}=\sup _{m \leq i \leq n-m+1}\left|F_{i}^{(r)}\right|$. Let $F_{(1)} \leq F_{(2)} \leq \cdots \leq F_{(B)}$ be the order statistics of $F_{r}$. Then $F_{\lfloor(1-\alpha) B\rfloor}$ consistently estimates the level $\alpha$ critical value for the gradient-based structural change test (21).

THEOREM 3.5. Under conditions of Theorem 3.3 and 3.4, Algorithm 3.1 generates consistent estimator of the level $\alpha$ critical value for the test (21).

In the Supplementary Material [29], we also extend our method to test structural changes for multiple M-estimators simultaneously and discuss the applicability of the method to dynamic models.

3.3. Bandwidth selection. To implement our testing procedure, one has to choose the tuning parameters $c_{n}$ and $m$ (except for least squares regression where only $m$ needs to be chosen). Due to the complex data structure, a robust bandwidth selection method which does not depend on specific forms of the data generating mechanisms is desired. To this end, for selecting proper $m$ of Theorem 3.4, we apply the method of minimum volatility (MV) suggested by [32] to $\tilde{\Psi}_{m, n}(t)$ in (24). The procedures are quite similar except that we replace unknown $\psi\left(e_{i}\right) \mathbf{x}_{i}$ with estimated $\psi\left(\hat{e}_{i, n}\right) \mathbf{x}_{i}$. Define

$$
\hat{\gamma}_{m}(r / n, s / n)=\sum_{i=1}^{r \wedge s}\left(\hat{\varpi}_{i, m}-\frac{m}{n} \hat{\varpi}_{n}\right)^{2} /(m(n-m+1)) .
$$

Calculate $\left\{\hat{\gamma}_{m_{j}}(r / n, r / n)\right\}_{r=1}^{n-m_{j}+1}$ for a grid of possible window sizes $m_{1} \leq \cdots \leq$ $m_{M}$. Write

$$
\operatorname{se}\left(\left\{\hat{\gamma}_{m_{j}}(r / n, r / n)\right\}_{j=a}^{b}\right)=\left(\frac{1}{b-a} \sum_{j=a}^{b}\left(\hat{\gamma}_{m_{j}}(r / n, r / n)-\overline{\hat{\gamma}}(r / n, r / n)\right)^{2}\right)^{1 / 2},
$$

where $\overline{\hat{\gamma}}(r / n, r / n)=\frac{1}{b-a+1} \sum_{j=a}^{b} \hat{\gamma}_{m_{j}}(r / n, r / n)$. Then we choose $m=m_{j}$ where $j=\operatorname{argmin}_{4 \leq j \leq 3}\left(\max _{1 \leq r \leq n-m_{M}+1} \operatorname{se}\left(\left\{\hat{\gamma}_{m_{j+k}}(r / n, r / n)\right\}_{k=-3}^{3}\right)\right)$. For more discussions about the "MV" method, see [22]. We also apply the MV method to the selection of the bandwidth $c_{n}$. Our procedure of selecting $c_{n}$ is as follows:

(i) Choose suitable end points $a_{1}<a_{2}$, such that the optimal $c_{n} \in I:=$ $\left[a_{1}, a_{2}\right]$.

(ii) Divide interval $I$ into $\bar{m}$, say $\bar{m}=99$ pieces. Specifically, let $h_{1}=a_{1}$, $h_{100}=a_{2}$, and $h_{k^{\prime}}=a_{1}+\left(k^{\prime}-1\right)\left(a_{2}-a_{1}\right) / 99,1 \leq k^{\prime} \leq 100$.

(iii) For each $h_{i}$, use it as a bandwidth to calculate the estimating quantity $\left\{\hat{\Lambda}_{h_{i}}\left(t_{j}\right)\right\}_{j=1}^{n}$. Let $C(i)$ be the maximum of RHS process of $(21)$ for $t \in\left[\frac{1}{n}, 1\right]$ obtained by replacing $\left\{U(t), t \in\left[\frac{1}{n}, 1\right]\right\}$ with $\left\{\sum_{i=1}^{\lfloor n t\rfloor} \frac{\psi\left(\hat{e}_{i, n}\right) \mathbf{x}_{i}}{\sqrt{n}}, t \in\left[\frac{1}{n}, 1\right]\right\}$ and replacing $\left\{\Lambda(t), t \in\left[\frac{1}{n}, 1\right]\right\}$ with $\left\{\hat{\Lambda}_{h_{i}}(t), t \in\left[\frac{1}{n}, 1\right]\right\}$, respectively. 
(iv) Define $D(s)=\left\{\frac{1}{2 k} \sum_{j=s-k}^{s+k}\left[C(j)-\frac{1}{2 k+1} \sum_{j=s-k}^{s+k} C(j)\right]^{2}\right\}^{1 / 2}$ for some $k>0$. Let $l$ be the minimizer of $\{D(s)\}_{s=k+1}^{100-k}$. Then we select $h_{l}$ as our bandwidth $c_{n}$.

Since $\Lambda(t)$ is a $p \times p$ matrix, directly applying the MV method for selecting $c_{n}$ will be time-consuming. Our proposed procedure is based on the fact that $U(t)$ is the limiting distribution of $\sum_{i=1}^{\lfloor n t\rfloor} \frac{\psi\left(e_{i, n}\right) \mathbf{x}_{i}}{\sqrt{n}}$. This motivates us to generate the pseudo limiting distribution via replacing $U(t)$ with $\sum_{i=1}^{\lfloor n t\rfloor} \frac{\psi\left(\hat{e}_{i, n}\right) \mathbf{x}_{i}}{\sqrt{n}}$ and choose the bandwidth as the one which stabilizes the pseudo quantity mostly. The numeric experiment shows that our bandwidth selection criteria work well under various circumstances.

\section{Simulation studies.}

4.1. Type I error. In this section, we examine the performance of our method for $\mathcal{L}_{2}$ (least squares) regression, the Huber regression with $\varsigma=1.5, \mathcal{L}_{1.5}$ regression and quantile regression with quantiles 0.5 and 0.8 . Throughout our simulations, the number of bootstrap samples $B=2000$. For quantile regression, we also compare our results with SQ method in [26]. The description of the SQ method for $\tau$ th quantile regression is as follows.

Let $\mathbf{X}=\left(\mathbf{x}_{1}, \ldots, \mathbf{x}_{n}\right)^{\prime}$. Define $H_{\lambda, n}(\hat{\beta})=\left(\mathbf{X X}^{\prime}\right)^{-1 / 2} \sum_{i=1}^{\lfloor\lambda n\rfloor} \mathbf{x}_{i} \psi_{\tau}\left(y_{i}-\mathbf{x}_{i}^{\prime} \hat{\beta}\right)$, where $\psi_{\tau}(x)=\tau-\mathbf{1}(x \leq 0)$. Then the SQ test statistic is defined as

$$
\mathrm{SQ}_{\tau}=\sup _{\lambda \in[0,1]}\left\|(\tau(1-\tau))^{-1 / 2}\left[H_{\lambda, n}(\hat{\beta})-\lambda H_{1, n}(\hat{\beta})\right]\right\|_{\infty} .
$$

The associated critical values for the $\mathrm{SQ}_{\tau}$ test are in Table 1 of [26]. To estimate $\{\Lambda(t), t \in(0,1]\}$, we choose bandwidth from 100 equally spaced points in a certain range. In each iteration, we select the bandwidths by the MV method we proposed in Section 3.3.

We consider the following heteroscedastic linear regression model:

$$
y_{i}=1+x_{i}+e_{i}, \quad e_{i}=\left(1+\gamma x_{i}\right)\left(\Upsilon_{i}-c_{i}\right) / 2
$$

for $i=1, \ldots, n, \gamma=0.1$. In our simulations, $x_{i}$ are i.i.d. $\chi^{2}(5) / 5$ and $c_{i}=\tilde{F}_{i}^{-1}(0)$ where $\tilde{F}_{i}(x)=\mathbb{E}\left(\psi\left(\Upsilon_{i}-x\right)\right)$. Let filtration $\mathcal{F}_{i}=\left(\varepsilon_{-\infty}, \ldots, \varepsilon_{i}\right)$ where $\left\{\varepsilon_{s}\right\}_{s=-\infty}^{\infty}$ are independent of $\left\{x_{i}\right\}_{i=1}^{n}$. We shall consider the following models for $\left\{\Upsilon_{i}\right\}_{i=-\infty}^{\infty}$ :

(I) Consider

$$
\Upsilon_{i}=G\left(t_{i}, \mathcal{F}_{i}\right), \quad G\left(t, \mathcal{F}_{i}\right)=0.75 \cos (2 \pi t) G\left(t, \mathcal{F}_{i-1}\right)+\varepsilon_{i},
$$

where $\varepsilon_{i}$ are i.i.d. $N(0,1)$. This is a locally stationary model since its AR(1) coefficient $0.75 \cos (2 \pi t)$ changes smoothly over $(0,1]$. 
(II) Consider $\Upsilon_{i}=G\left(t_{i}, \mathcal{F}_{i}\right)$, and

$$
G\left(t, \mathcal{F}_{i}\right)=G_{1}\left(t, \mathcal{F}_{i}\right) \mathbf{1}(0<t \leq 0.8)+G_{2}\left(t, \mathcal{F}_{i}\right) \mathbf{1}(0.8<t \leq 1),
$$

where

$$
\begin{aligned}
& G_{1}\left(t, \mathcal{F}_{i}\right)=0.6 \cos (2 \pi t) G_{1}\left(t, \mathcal{F}_{i-1}\right)+\varepsilon_{i}, \\
& G_{2}\left(t, \mathcal{F}_{i}\right)=(0.5-t) G_{2}\left(t, \mathcal{F}_{i-1}\right)+\varepsilon_{i}
\end{aligned}
$$

and $\varepsilon_{i}$ 's are i.i.d. $N(0,1)$. This is a PLS model. The AR coefficient changes smoothly before and after $t=0.8$, with an abrupt change at $t=0.8$.

( $\left.\mathrm{II}^{\prime}\right)$ Model $\mathrm{II}^{\prime}$ is the same as model II except we change the i.i.d. $N(0,1) \varepsilon_{i}$ 's to i.i.d. student $t$ distribution with 12 degrees of freedom [ $t$ (12)].

(II*) Model II* is the same as model $\mathrm{II}^{\prime}$ except we set $x_{i}$ i.i.d. $1+(5 / 3)^{-1 / 2} t(5)$.

Note that in Models I, II, II' and II*, the covariates are independent and identically distributed. However, the errors are heteroscedastic with respect to the covariates. Furthermore, the errors are PLS processes which exhibit smooth and (or) abrupt changes in their data generating mechanisms over time.

We also consider the following nonstationary dynamic model III. Let $\left\{z_{i}\right\}_{i \in \mathbb{Z}}$ be i.i.d. $\chi^{2}(1)$ random variables, and

(III) $y_{i}=0.3 y_{i-1} /\left(1+z_{i-1}\right)+e_{i} / 3, y_{0} \sim N(0,1)$, where $e_{i}=G\left(t_{i}, \mathcal{F}_{i}\right)$ with

$$
\begin{aligned}
G\left(t, \mathcal{F}_{i}\right)= & \left(1+\frac{1}{3}(t-1)^{2}\right) \epsilon_{i} \mathbf{1}(0<t \leq 0.5) \\
& +(1+0.5 \cos (2 \pi t)) \epsilon_{i} \mathbf{1}(0.5<t \leq 1), \quad \epsilon_{i} \text { 's i.i.d. } N(0,1),
\end{aligned}
$$

Finally, we consider the following scenario IV. Let $\Upsilon_{i}$ be the PLS process defined in Model II and $c_{i}$ be the corresponding quantity defined below (25). Let $\left\{\varepsilon_{i}\right\}_{i \in \mathbb{Z}},\left\{\eta_{i}\right\}_{i \in \mathbb{Z}},\left\{\epsilon_{i}\right\}_{i \in \mathbb{Z}}$ be i.i.d. $N(0,1)$ 's. Furthermore, $\left\{\varepsilon_{i}\right\}_{i \in \mathbb{Z}},\left\{\eta_{i}\right\}_{i \in \mathbb{Z}}$ and $\left\{\epsilon_{i}\right\}_{i \in \mathbb{Z}}$ are independent of each other. Let $v_{i}=\frac{\eta_{i}+\epsilon_{i}}{\sqrt{2}}$. Consider:

(IV) Let $x_{1, i}$ be the PLS process generated from $G_{1}\left(t, \mathcal{G}_{i}\right)=\sum_{j=0}^{\infty}(0.5-$ $0.5 t)^{j} v_{i-j}$, and $x_{2, i}$ be the PLS process generated from $G_{2}\left(t, \mathcal{G}_{i}\right)=\sum_{j=0}^{\infty}(0.25+$ $0.5 t)^{j} \epsilon_{i-j}$, where $\mathcal{G}_{i}=\left(\ldots, \epsilon_{i}, \eta_{i}\right)$. Let

$$
y_{i}=1+x_{1, i}+x_{2, i}+e_{i}, \quad e_{i}=\frac{\sqrt{1+x_{1, i}+x_{2, i}}\left(\Upsilon_{i}-c_{i}\right)}{4} .
$$

Note that in Model IV both the covariates and errors are nonstationary time series. We examine our test with sample sizes 300 and 600 at two nominal levels $5 \%$ and $10 \%$. We report the simulated type I errors in Tables 1-2 for our proposed gradient-vector-based test (21). In Table 1, we present our simulation results for the Huber regression, $\mathcal{L}_{1.5}$ regression and least squares regression, respectively. Table 2 contains our simulation results for quantile regressions with $\tau=0.5$ and 
TABLE 1

Simulated type I error rates for Huber $(\varsigma=1.5), \mathcal{L}_{1.5}$ and $\mathcal{L}_{2}$ regression

\begin{tabular}{|c|c|c|c|c|c|c|c|c|c|c|c|c|}
\hline & & Iuber $r$ & gressi & & & $\mathcal{L}_{1.5} \mathrm{re}$ & ressio & & Le: & it squar & s regr & ssion \\
\hline & & 300 & & 600 & & 300 & & 600 & & 300 & & 600 \\
\hline & $5 \%$ & $10 \%$ & $5 \%$ & $10 \%$ & $5 \%$ & $10 \%$ & $5 \%$ & $10 \%$ & $5 \%$ & $10 \%$ & $5 \%$ & $10 \%$ \\
\hline I & 4.25 & 11.55 & 4.35 & 11 & 5.3 & 13.05 & 4.8 & 11.55 & 5.55 & 13.2 & 5.35 & 12.25 \\
\hline II & 4.45 & 10.35 & 4.55 & 9.95 & 3.85 & 9.8 & 4.25 & 10.4 & 4.8 & 12.05 & 5.2 & 11.6 \\
\hline $\mathrm{II}^{\prime}$ & 4.1 & 10.35 & 4.75 & 10.3 & 4.35 & 10.3 & 4.7 & 10.2 & 5.65 & 12.35 & 4.8 & 10.9 \\
\hline $\mathrm{II}^{*}$ & 4.05 & 9.95 & 5.2 & 10.75 & 3.7 & 9.25 & 4.2 & 10.05 & 4.9 & 11.7 & 4.2 & 10.65 \\
\hline III & 4.3 & 10.55 & 4.95 & 10.85 & 4.3 & 10.85 & 4.65 & 10.3 & 3.95 & 10.6 & 4.45 & 10.15 \\
\hline IV & 3.15 & 8.0 & 5.1 & 10.85 & 3.9 & 8.75 & 4.75 & 10.25 & 3.15 & 7.95 & 4.4 & 9.6 \\
\hline
\end{tabular}

0.8 , respectively. The simulated Type I errors are quite close to the nominal levels. Meanwhile, our simulation results show that increasing the sample size from 300 to 600 in general significantly improves the performance of our test. In addition, for quantile regression, the test performs better when the quantile is less extreme. The Monte Carlo experiments in Table 3 also show the inadequacy of the SQ method when $e_{i}$ and (or) $x_{i}$ are nonstationary. For comparison purposes, we also generated a stationary $\operatorname{AR}(0.5)$ model: $y_{i}=0.5 y_{i-1}+\varepsilon_{i}$ where $\varepsilon_{i}$ 's are i.i.d. $N(0,1)$. We find that the SQ method works well for this stationary $\operatorname{AR}(0.5)$ model, which is consistent with the results reported in [26]. Meanwhile, simulation results show that our method performs almost as well as SQ method under the stationary scenario.

TABLE 2

Simulated type I error rates for quantile regressions, gradient-based test

\begin{tabular}{|c|c|c|c|c|c|c|c|c|}
\hline & \multicolumn{4}{|c|}{0.5 quantile } & \multicolumn{4}{|c|}{0.8 quantile } \\
\hline & \multicolumn{2}{|c|}{$n=300$} & \multicolumn{2}{|c|}{$n=600$} & \multicolumn{2}{|c|}{$n=300$} & \multicolumn{2}{|c|}{$n=600$} \\
\hline & $5 \%$ & $10 \%$ & $5 \%$ & $10 \%$ & $5 \%$ & $10 \%$ & $5 \%$ & $10 \%$ \\
\hline Model I & 4.8 & 14.05 & 4.1 & 10.9 & 4.8 & 13.45 & 4.55 & 10.75 \\
\hline Model II & 4.05 & 11.35 & 4.25 & 11.1 & 4.95 & 12.55 & 4.35 & 9.95 \\
\hline Model II' & 4.7 & 12.05 & 4.25 & 10.6 & 5.6 & 12.3 & 4.65 & 10.9 \\
\hline Model II* & 3.1 & 10.1 & 3.65 & 9.05 & 3.6 & 10.75 & 3.8 & 9.65 \\
\hline Model III & 4.35 & 9.2 & 5.2 & 10.3 & 3.95 & 10.2 & 4.3 & 10.15 \\
\hline Model IV & 4.0 & 8.55 & 4.25 & 9.45 & 3.65 & 10.1 & 4.5 & 10.2 \\
\hline $\mathrm{AR}(0.5)$ & 4.05 & 9.2 & 4.95 & 10.85 & 4.2 & 9.25 & 5.55 & 10.25 \\
\hline
\end{tabular}


TABLE 3

Simulated type I error rates for quantile regressions, $S Q$ method

\begin{tabular}{|c|c|c|c|c|c|c|c|c|}
\hline & \multicolumn{4}{|c|}{0.5 quantile } & \multicolumn{4}{|c|}{0.8 quantile } \\
\hline & \multicolumn{2}{|c|}{$n=300$} & \multicolumn{2}{|c|}{$n=600$} & \multicolumn{2}{|c|}{$n=300$} & \multicolumn{2}{|c|}{$n=600$} \\
\hline & $5 \%$ & $10 \%$ & $5 \%$ & $10 \%$ & $5 \%$ & $10 \%$ & $5 \%$ & $10 \%$ \\
\hline I & 15 & 24.8 & 15.65 & 24.85 & 11.3 & 19.1 & 14.9 & 24.2 \\
\hline II & 5.55 & 10.55 & 5.55 & 11.45 & 5.15 & 10.8 & 6.55 & 12.7 \\
\hline $\mathrm{II}^{\prime}$ & 5.7 & 10.25 & 6 & 10.8 & 5.2 & 10.8 & 6.05 & 12.2 \\
\hline II* & 4.8 & 9.3 & 5.9 & 11.75 & 6.75 & 12.15 & 7.1 & 13.3 \\
\hline III & 2.25 & 4.7 & 3.1 & 6.15 & 5.7 & 10.8 & 6.85 & 13.05 \\
\hline IV & 9.15 & 18.75 & 9.5 & 19.65 & 9.05 & 17.9 & 9.9 & 20.2 \\
\hline $\mathrm{AR}(0.5)$ & 5.4 & 10.45 & 4.65 & 10.1 & 3.85 & 7.55 & 4.85 & 9.45 \\
\hline
\end{tabular}

4.2. Simulated power. We consider the alternative model that

$$
y_{i}=1+x_{i}(1+\delta \mathbf{1}(i \geq\lfloor n / 2\rfloor))+e_{i}, \quad e_{i}=\left(1+\gamma x_{i}\right)\left(\Upsilon_{i}-c_{i}\right) / 2,
$$

where $\Upsilon_{i}$ follows model II, which is PLS. We shall simulate different jump sizes $\delta$ to investigate the power performances of our testing procedures. The sample size and significance level are 300 and $10 \%$ in our simulation. The left panel of Figure 1 examines the simulated powers for the Huber regression with $\varsigma=1.5, \mathcal{L}_{1.5}$ regression, $\mathcal{L}_{2}$ (least squares) regression and median qunatile regression. The results show that our testing procedure has decent power for general M-estimation with moderate sample size. As expected, the regression with more robust loss function tends to have less power. The significance level is $10 \%$. We also construct and examine a regression-coefficient-based CUSUM test and find that it has a significantly inferior power performance than the gradient-based test. See the right panel of Figure 1. Additional empirical results show that under stationarity, our method is a little less powerful than the SQ method in [26] when investigating quantile regression but more powerful than the SCB method in [33] when investigating least squares regression. The detailed results and explanations are relegated to Section 5 of the online Supplementary Material [29].

5. Data analysis. In this section, we apply our method to the Hong Kong circulatory and respiratory data. It consists of daily measurements of pollutants and daily hospital admissions in Hong Kong between January 1, 1994, and December 31, 1995. This dataset has been analyzed under i.i.d. assumptions in [13, 14] and [10] among others. It has also been studied under locally stationary assumptions; see, for example, [33] and [31]. The aim of this data analysis is to capture the relationship between the daily total number of hospital admissions of circulation/respiration and the levels of pollutants such as sulphur dioxide $\left(\mathrm{SO}_{2}\right)$ (in micrograms per cubic metre), nitrogen dioxide $\left(\mathrm{NO}_{2}\right)$ (in micrograms per cubic 

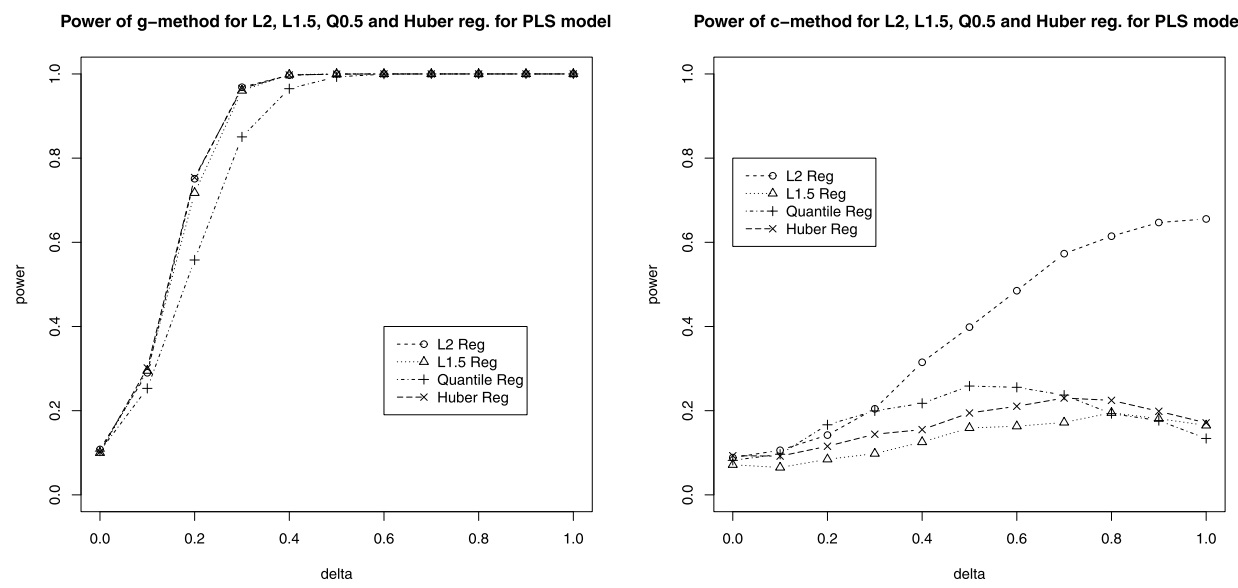

FIG. 1. Simulated power with $\Upsilon_{i}$ following Model II for gradient-based method (left) and for coefficient-based method (right).

metre) and dust (in micrograms per cubic metre). By fitting time-varying linear regression models, the results of [33] indicated the existence of change points in the least squares regression coefficients between January 1, 1994, and December 31,1995 . By carefully observing the patterns of regression coefficients and their simultaneous confidence band plotted in Figure 2 of their paper, it is difficult to tell whether there is a change point in the mean regression relationship between January 1, 1995, and December 31, 1995. To justify the necessity to use the PLS formulation of the errors and covariates, we first perform the tests in [32] and [11] for change points in the mean and autocovariances. The $p$-values for no change point in mean for $\mathrm{NO}_{2}, \mathrm{SO}_{2}$ and dust are $0.5 \%, 5.15 \%$ and $0.025 \%$, respectively. For $\mathrm{NO}_{2}$ and dust, the $p$-values for no change points in lag-1 autocovariance are $9.9 \%$ and $8 \%$, respectively, while the $p$-values for no change points in lag-2 autocovariance are $4.7 \%$ and $3.3 \%$, respectively. These results show strong evidence that the data we considered are nonstationary. Thus, we consider the following model:

$$
y_{i}=\beta_{0}+\sum_{l=1}^{3} \beta_{l} x_{i, l}+\varepsilon_{i},
$$

where $y_{i}$ is the daily number of hospital admissions, and $\left\{x_{i, 1}, 1 \leq i \leq n\right\}$ is the level of $\mathrm{SO}_{2},\left\{x_{i, 2}, 1 \leq i \leq n\right\}$ is the level of $\mathrm{NO}_{2},\left\{x_{i, 3}, 1 \leq i \leq n\right\}$ is the level of dust, and $\varepsilon_{i}$ is a PLS noise. We test the null hypothesis that $\beta:=\left(\beta_{0}, \ldots, \beta_{3}\right)^{\prime}$ remains constant from January 1, 1995, to December 31, 1995, for both least squares regression and quantile regression. For quantile regression, we consider 7 different quantiles $0.2,0.3,0.4,0.5,0.6,0.7,0.8$. For the least squares regression, we choose bandwidth $m=20$. It turns out that the $90 \%$ critical value is $10,532.89$, 
TABLE 4

Structural Change Test for 0.2, 0.3, 0.4, 0.5, 0.6, 0.7, 0.8 quantiles. The null hypothesis of no structural change in the relationship between daily hospital admissions and pollutants levels is rejected for 0.2, 0.3, 0.4 quantiles at 5\% significance level, rejected for 0.5 quantile at $10 \%$ significance level, and is not rejected for 0.6, 0.7, 0.8 quantiles

\begin{tabular}{lccccccc}
\hline Quantile & $\mathbf{0 . 2 *}$ & $\mathbf{0 . 3}$ & $\mathbf{0 . 4}$ & $\mathbf{0 . 5}^{* *}$ & $\mathbf{0 . 6}$ & $\mathbf{0 . 7}$ & $\mathbf{0 . 8}$ \\
\hline Test statistics & \multicolumn{1}{c}{61.27} & 79.74 & 85.53 & 81.84 & 74.52 & 78 & 70.27 \\
$m$ & 13 & 12 & 7 & 12 & 12 & 12 & 17 \\
h & 0.84 & 0.67 & 0.16 & 0.36 & 0.26 & 0.16 & 0.27 \\
$90 \%$ & 48.65 & 68.34 & 74.75 & 75.16 & 96.39 & 104.7 & 102.83 \\
$95 \%$ & 53.83 & 78.23 & 85.33 & 82.92 & 112.42 & 120.74 & 115.41 \\
SQ method & 5.38 & 5.86 & 6.19 & 5.85 & 5.32 & 6.28 & 6.8 \\
\hline
\end{tabular}

and $95 \%$ critical value is $11,973.6$, while the test statistic is $10,464.35$. Our result shows that there is no evidence indicating that the relationship in mean between hospital admissions and pollutants changed in the year of 1995 under the PLS assumptions.

We also summarize the quantile regression results in Table 4.

Our results show that for mid and low quantiles there are structural changes in the regression coefficients; while for high quantiles there are none. For 0.5 quantile, our results show that the $p$-value is between $5 \%$ and $10 \%$. This is a potentially interesting finding which shows the influence of pollutants on low hospital admissions has changed while there is no change in the relationship on the high end. Consequently, it is appropriate to use a parametric model to fit the high quantile regression while nonparametric dynamic models are more appropriate to model the low quantiles. Such asymmetric behavior across different quantiles cannot be identified by the hypothesis testing procedures in mean regression proposed in [31]. The last line of the table lists the test statistics generated via SQ method. The $95 \%$ and $99 \%$ critical values of the SQ test are 1.569 and 1.795, respectively. By Table 4, the SQ method strongly rejects the null hypothesis at all quantiles considered in this paper. This is likely due to the violation of the strict stationarity assumptions in [26] for this data set, which makes SQ test overact to the spurious patterns of change points in regression coefficients caused by the nonstationary errors and covariates. As a result, it seems that the SQ method in [26] cannot detect the asymmetric behavior across different quantiles and it yields too significant testing results with too small $p$-values due to the nonstationarity of the errors and covariates of the regression.

Acknowledgments. We thank two Editors, an Associate Editor and three referees for their insightful and constructive comments which greatly improve the quality and presentation of the paper. 


\section{SUPPLEMENTARY MATERIAL}

\section{Supplement to "Gradient-based structural change detection for nonsta-} tionary time series M-estimation”, (DOI: 10.1214/17-AOS1582SUPP; .pdf). We provide (a) detailed proofs of the theorems and lemmas, (b) theoretical investigation on parameter estimation under the alternative hypothesis, (c) the analysis of dynamic models, (d) extension of our methodology to finitely many M-estimations and (e) extra simulation results.

\section{REFERENCES}

[1] ANDREws, D. W. K. (1993). Tests for parameter instability and structural change with unknown change point. Econometrica 61 821-856. MR1231678

[2] Arcones, M. A. (1996). The Bahadur-Kiefer representation of $L_{p}$ regression estimators. Econometric Theory 12 257-283. MR1395032

[3] Aue, A. and Horváth, L. (2013). Structural breaks in time series. J. Time Series Anal. 34 1-16. MR3008012

[4] BABU, G. J. (1989). Strong representations for LAD estimators in linear models. Probab. Theory Related Fields 83 547-558. MR1022629

[5] Bahadur, R. R. (1966). A note on quantiles in large samples. Ann. Math. Stat. 37 577-580. MR0189095

[6] BAI, J. (1996). Testing for parameter constancy in linear regressions: An empirical distribution function approach. Econometrica 64 597-622. MR1385559

[7] BAI, J. and PERRON, P. (1998). Estimating and testing linear models with multiple structural changes. Econometrica 66 47-78. MR1616121

[8] Bollerslev, T. (1986). Generalized autoregressive conditional heteroskedasticity. J. Econometrics 31 307-327. MR0853051

[9] Brown, R. L., Durbin, J. and Evans, J. M. (1975). Techniques for testing the constancy of regression relationships over time. J. Roy. Statist. Soc. Ser. B 37 149-192. MR0378310

[10] CAI, Z., FAN, J. and LI, R. (2000). Efficient estimation and inferences for varying-coefficient models. J. Amer. Statist. Assoc. 95 888-902. MR1804446

[11] Dette, H., Wu, W. and Zhou, Z. (2015). Change point analysis of second order characteristics in non-stationary time series. Available at arXiv:1503.08610.

[12] ENGLE, R. F. (1982). Autoregressive conditional heteroscedasticity with estimates of the variance of United Kingdom inflation. Econometrica 50 987-1007. MR0666121

[13] FAN, J. and ZHANG, W. (1999). Statistical estimation in varying coefficient models. Ann. Statist. 27 1491-1518. MR1742497

[14] FAn, J. and ZhANG, W. (2000). Simultaneous confidence bands and hypothesis testing in varying-coefficient models. Scand. J. Stat. 27 715-731. MR1804172

[15] Hansen, B. E. (2000). Testing for structural change in conditional models. J. Econometrics 97 93-115. MR1788819

[16] HE, X. and ZHU, L.-X. (2003). A lack-of-fit test for quantile regression. J. Amer. Statist. Assoc. 98 1013-1022. MR2041489

[17] JuHL, T. and XIAO, Z. (2009). Tests for changing mean with monotonic power. J. Econometrics 148 14-24. MR2494814

[18] KIEFER, J. (1967). On Bahadur's representation of sample quantiles. Ann. Math. Stat. 381323 1342. MR0217844

[19] Lahiri, S. N. (2003). Resampling Methods for Dependent Data. Springer, New York. MR2001447 
[20] Mccabe, B. P. M. and Harrison, M. J. (1980). Testing the constancy of regression relationships over time using least squares residuals. J. R. Stat. Soc. Ser. C. Appl. Stat. 29142 148.

[21] Ploberger, W. and KräMer, W. (1992). The CUSUM test with OLS residuals. Econometrica 60 271-285. MR1162619

[22] Politis, D. N., Romano, J. P. and Wolf, M. (1999). Subsampling. Springer, New York. MR1707286

[23] Portnoy, S. (1991). Asymptotic behavior of regression quantiles in nonstationary, dependent cases. J. Multivariate Anal. 38 100-113. MR1128939

[24] Powell, J. L. (1991). Estimation of monotonic regression models under quantile restrictions. In Nonparametric and Semiparametric Methods in Econometrics and Statistics (Durham, NC, 1988). Internat. Sympos. Econom. Theory Econometrics 357-384. Cambridge Univ. Press, Cambridge. MR1174980

[25] PRÁšKovÁ, Z. and ChOChOlA, O. (2014). M-procedures for detection of a change under weak dependence. J. Statist. Plann. Inference 149 60-76. MR3199894

[26] QU, Z. (2008). Testing for structural change in regression quantiles. J. Econometrics 146170 184. MR2459652

[27] SU, L. and White, H. (2010). Testing structural change in partially linear models. Econometric Theory 26 1761-1806. MR2738016

[28] Tong, H. (1990). Nonlinear Time Series: A Dynamical System Approach. Oxford Statistical Science Series 6. Oxford Univ. Press, New York. MR1079320

[29] WU, W. and ZHOU, Z. (2018). Supplement to "Gradient-based structural change detection for nonstationary time series M-estimation." DOI:10.1214/17-AOS1582SUPP.

[30] WU, W. B. (2007). M-estimation of linear models with dependent errors. Ann. Statist. 35 495521. MR2336857

[31] ZHANG, T. and WU, W. B. (2012). Inference of time-varying regression models. Ann. Statist. 40 1376-1402. MR3015029

[32] ZHOU, Z. (2013). Heteroscedasticity and autocorrelation robust structural change detection. J. Amer. Statist. Assoc. 108 726-740. MR3174655

[33] ZHOU, Z. and WU, W. B. (2010). Simultaneous inference of linear models with time varying coefficients. J. R. Stat. Soc. Ser. B. Stat. Methodol. 72 513-531. MR2758526

DEPARTMENT OF STATISTICS

UNIVERSITY COLLEGE

GOWER STREET

WC1E 6BT, LONDON

UNITED KINGDOM

E-MAIL:w.wu@ucl.ac.uk
Department of Statistical Science UNIVERSITY OF TORONTO

100 St. GEORGE ST.

TORONTO, ONTARIO M5S 3G3

CANADA

E-MAIL: zhou@utstat.toronto.edu 